\title{
Successive Refinement of Abstract Sources
}

\author{
Victoria Kostina, Ertem Tuncel
}

\begin{abstract}
In successive refinement of information, the decoder refines its representation of the source progressively as it receives more encoded bits. The rate-distortion region of successive refinement describes the minimum rates required to attain the target distortions at each decoding stage. In this paper, we derive a parametric characterization of the rate-distortion region for successive refinement of abstract sources. Our characterization extends Csiszár's result [2] to successive refinement, and generalizes a result by Tuncel and Rose [3], applicable for finite alphabet sources, to abstract sources. This characterization spawns a family of outer bounds to the rate-distortion region. It also enables an iterative algorithm for computing the rate-distortion region, which generalizes Blahut's algorithm to successive refinement. Finally, it leads a new nonasymptotic converse bound. In all the scenarios where the dispersion is known, this bound is secondorder optimal.

In our proof technique, we avoid Karush-Kuhn-Tucker conditions of optimality, and we use basic tools of probability theory. We leverage the Donsker-Varadhan lemma for the minimization of relative entropy on abstract probability spaces.
\end{abstract}

Index Terms-Successive refinement, rate-distortion theory, single-shot analysis, d-tilted information, Blahut algorithm, converse, dispersion.

\section{INTRODUCTION}

For a source random variable $X \in \mathcal{X}$ and a distortion measure $\mathrm{d}: \mathcal{X} \times \mathcal{Y} \mapsto[0,+\infty)$, where $\mathcal{X}$ and $\mathcal{Y}$ are abstract sets (source and reproduction alphabets), the classical informational rate-distortion function is defined as the following minimal mutual information quantity:

$$
R(d) \triangleq \inf _{\substack{P_{Y \mid X}: \mathcal{X} \mapsto \mathcal{Y} \\ \mathbb{E}[\mathrm{d}(X, Y)] \leq d}} I(X ; Y)
$$

This convex optimization problem rarely has an explicit solution. The following result provides a parametric representation:

Theorem 1 (Parametric representation of $R(d)$ [2]). Assume that the following conditions are met.

(A) $d_{\min }<d<d_{\max }$, where

$$
\begin{aligned}
& d_{\min } \triangleq \inf \{d: \quad R(d)<\infty\} \\
& d_{\max } \triangleq \inf \left\{d: \quad R(d) \text { is constant on }\left(d_{\max }, \infty\right)\right\}
\end{aligned}
$$

(B) There exists a transition probability kernel $P_{Y^{\star} \mid X}$ that attains the infimum in (1).

Then, it holds that

$$
R(d)=\max _{\alpha(x), \lambda}\{-\mathbb{E}[\log \alpha(X)]-\lambda d\}
$$

V. Kostina (e-mail: vkostina@caltech.edu) is with California Institute of Technology. E. Tuncel (e-mail: ertem.tuncel@ucr.edu) is with University of California, Riverside. This work was supported in part by the National Science Foundation (NSF) under Grant CCF-1566567. It was presented in part at ISIT 2017 [1]. where the maximization is over $\alpha(x) \geq 0$ and $\lambda \geq 0$ satisfying the constraint

$$
\mathbb{E}\left[\frac{\exp (-\lambda \mathrm{d}(X, y))}{\alpha(X)}\right] \leq 1 \forall y \in \mathcal{Y} .
$$

Furthermore, in order for $P_{Y^{\star} \mid X}$ to achieve the infimum in (1), it is necessary and sufficient that

$$
\frac{d P_{X \mid Y^{\star}=y}}{d P_{X}}(x)=\frac{\exp \left(-\lambda^{\star} \mathrm{d}(x, y)\right)}{\alpha(x)},
$$

where $^{1}$

$$
\lambda^{\star}=-R^{\prime}(d)
$$

and $0 \leq \alpha(x) \leq 1$ satisfies (5). Finally, the choice

$$
\alpha^{\star}(x)=\mathbb{E}\left[\exp \left(-\lambda^{\star} \mathrm{d}\left(x, Y^{\star}\right)\right)\right],
$$

satisfies both (5) and (6); thus $\left(\alpha^{\star}(x), \lambda^{\star}\right)$ is the maximizer of (4).

In (6), $\frac{d P}{d Q}$ denotes the Radon-Nykodym derivative; if $P$ and $Q$ are both discrete / continuous probability distributions, $\frac{d P}{d Q}$ is simply the ratio of corresponding probability mass / density functions. Theorem 1 applies to the much more general setting of abstract probability spaces. It was Csiszár [2] who formulated and proved Theorem 1 in this generality. ${ }^{2}$ For finite alphabet sources, the parametric representation of $R(d)$ is contained in Shannon's paper [4]; Gallager's [5, Th. 9.4.1] and Berger's [6] texts include the parametric representation of $R(d)$ for discrete and continuous sources. Csiszár and Körner's book [7, Th. 8.7] presents a derivation of the parametric representation of the discrete rate-distortion function that employs variational principles.

The parametric representation of $R(d)$ plays a key role in the Blahut algorithm [8] for computing the rate-distortion function. For difference distortion measures, $\mathrm{d}(x, y)=\mathrm{d}(x-y)$, a certain choice of $(\alpha(x), \lambda)$ in (4) leads to the Shannon lower bound [4], a particularly simple, explicit lower bound to the rate-distortion function, which offers nice intuitions and which is known to be tight in the limit $d \downarrow 0$. Leveraging Theorem 1, a generalization of Shannon's lower bound to abstract probability spaces was recently proposed [9], [10].

\footnotetext{
${ }^{1}$ The differentiability of $R(d)$ is assured by the assumptions that the distortion measure $d$ cannot take the value $+\infty$ and that there exists a $P_{Y \star \mid X}$ attaining the infimum in (1) [2, p. 69]. If we allow $d$ to take the value $+\infty$, then it is possible that $R(d)$ is not differentiable at some $d$. In that case, Theorem 1 will hold verbatim replacing $\lambda^{\star}$ by the negative slope of any tangent to $R(d)$ at $d$. With this easy extension in mind, we choose to limit our attention to finite-valued distortion measures to ensure differentiability. Note also that while $P_{Y^{\star} \mid X}$ need not be unique, $\alpha^{\star}(x)$ is (and therefore, through (6), so is $P_{X^{\star} \mid Y}$ ); this is a consequence of differentiability of $R(d)$ [2, p. 69].

${ }^{2}$ Even more generally, Csiszár [2] showed that (4) continues to hold even if the infimum in (1) is not attained by any conditional probability distribution.
} 
Furthermore, given $\left(P_{X}, \mathrm{~d}\right)$, the d-tilted information, defined for each realization $x \in \mathcal{X}$ through the solution to (4) as

$$
\jmath_{\mathrm{d}}(x, d) \triangleq-\log \alpha^{\star}(x)-\lambda^{\star} d,
$$

governs the nonasymptotic fundamental limits of lossy compression [11], where the subscript $d$ emphasizes the distortion measure used.

In this paper, we state and prove a generalization of Theorem 1 to successive refinement of abstract alphabet sources. If the source is successively refinable, that is, if optimal successive coding achieves the respective rate-distortion functions at each decoding stage, our result recovers the representation in Theorem 1. Our characterization refines a prior finite alphabet result by Tuncel and Rose [3, Theorem 4] and extends it to abstract probability spaces. Our general setting necessitates the use of the mathematical tools fundamentally different from the standard convex optimization tools (Karush-KuhnTucker conditions) that can be used to solve the finite alphabet case, as carried out in [3]. We leverage the Donsker-Varadhan characterization of the minimum relative entropy, and, to show the necessary optimality conditions, we compare a tentative solution to a perturbation by a carefully selected auxiliary distribution.

The new characterization of rate-distortion function for successive refinement on abstract alphabets allows us to identify the key random variable describing the nonasymptotic fundamental limits of successive refinement, and to show a new nonasymptotic converse bound. In all the scenarios where the dispersion of successive refinement is known [12], [13], this bound is second-order optimal.

The new characterization also enables an iterative algorithm, which can be used to compute an accurate approximation to the rate-distortion function of successive refinement, even if the source and reproduction alphabets are not discrete. We prove that when initialized appropriately, the algorithm converges to the true value of rate-distortion function with speed $O\left(\frac{1}{k}\right)$, where $k$ is the iteration number. The algorithm can be viewed as a generalization of Blahut's algorithm [8] and its extension to successive refinement by Tuncel and Rose [3] for discrete alphabets. Methods to compute the capacity and rate-distortion functions for continuous alphabets were proposed in [14] and [15].

The rest of the paper is organized as follows. The main result of the paper characterizing the abstract rate-distortion function (Theorem 2) is presented in Section II. The main nonasymptotic converse result, Theorem 3, is shown in Section III. A proof of Theorem 1, which streamlines Csiszár's argument [2], is presented in Section IV. The proof of Theorem 2, which leverages the ideas presented in Section IV and in [3], is presented in Section V. Section VI discusses the iterative algorithm for computation of rate-distortion function of successive refinement.

Throughout the paper, $\mathbb{R}_{+}=[0,+\infty)$ is the positive real line; $P_{X}$-a.e. $x$ stands for 'almost every $x$ ', i.e. 'except on a set with total $P_{X}$ measure 0'; $P_{X} \rightarrow P_{Y \mid X} \rightarrow P_{Y}$ signifies that $P_{Y}$ is the distribution observed at the output of random transformation $P_{Y \mid X}$ when the input is distributed according to $P_{X}$, i.e. $P_{Y}$ is the marginal of $P_{X} P_{Y \mid X}$. When we say that a random variable $X$ takes values in a set $\mathcal{X}$, we understand that $\mathcal{X}$ comes together with its $\sigma$-algebra $\mathcal{X}$, forming a measurable space $(\mathcal{X}, \mathcal{X})$. Throughout the paper, we assume that all $\sigma$ algebras contain singletons (this is true for any countably separated $\sigma$-algebra). For two measurable spaces $(\mathcal{X}, \mathcal{X})$ and $(\mathcal{Y}, \mathcal{Y})$, a transition probability kernel from $(\mathcal{X}, \mathcal{X})$ into $(\mathcal{Y}, \mathcal{Y})$ is a mapping $\kappa: \mathcal{X} \times \mathcal{Y} \mapsto[0,1]$ such that $(i)$ the mapping $x \mapsto \kappa(x, B)$ is $\mathcal{X}$-measurable for every $B \in \mathcal{Y}$, and (ii) the mapping $B \mapsto \kappa(x, B)$ is a probability measure on $(\mathcal{Y}, y)$ for every $x \in \mathcal{X}$.

\section{Characterization OF RATE-Distortion FUnCTION}

Consider the source random variable $X \in \mathcal{X}$ and two (possibly different) distortion measures $\mathrm{d}_{1}: \mathcal{X} \times \mathcal{Y}_{1} \mapsto[0,+\infty)$ and $\mathrm{d}_{2}: \mathcal{X} \times \mathcal{Y}_{2} \mapsto[0,+\infty)$, quantifying the accuracy of lossy compression at the first and the second stages, respectively. An $\left(M_{1}, M_{2}, d_{1}, d_{2}\right)$ average distortion code for $\left(P_{X}, \mathrm{~d}_{1}, \mathrm{~d}_{2}\right)$ is a pair of encoders

$$
\begin{aligned}
& \mathrm{f}_{1}: \mathcal{X} \mapsto\left\{1, \ldots, M_{1}\right\} \\
& \mathrm{f}_{2}: \mathcal{X} \mapsto\left\{1, \ldots,\left\lfloor M_{2} / M_{1}\right\rfloor\right\}
\end{aligned}
$$

and decoders

$$
\begin{aligned}
& \mathrm{g}_{1}:\left\{1, \ldots, M_{1}\right\} \mapsto \mathcal{Y}_{1} \\
& \mathrm{~g}_{2}:\left\{1, \ldots, M_{1}\right\} \times\left\{1, \ldots,\left\lfloor M_{2} / M_{1}\right\rfloor\right\} \mapsto \mathcal{Y}_{2}
\end{aligned}
$$

such that

$$
\begin{aligned}
\mathbb{E}\left[\mathrm{d}_{1}\left(X, \mathrm{~g}_{1}\left(\mathrm{f}_{1}(X)\right)\right)\right] & \leq d_{1}, \\
\mathbb{E}\left[\mathrm{d}_{2}\left(X, \mathrm{~g}_{2}\left(\mathrm{f}_{1}(X), \mathrm{f}_{2}(X)\right)\right)\right] & \leq d_{2} .
\end{aligned}
$$

For the successive refinement of $n$ i.i.d. copies of $X$ with separable distortion measures $\mathrm{d}_{1}^{(n)}\left(x^{n}, y^{n}\right)=\frac{1}{n} \sum_{i=1}^{n} \mathrm{~d}_{1}\left(x_{i}, y_{i}\right)$, $\mathrm{d}_{2}^{(n)}\left(x^{n}, y^{n}\right)=\frac{1}{n} \sum_{i=1}^{n} \mathrm{~d}_{2}\left(x_{i}, y_{i}\right)$, we say that the distortions $\left(d_{1}, d_{2}\right)$ are asymptotically attainable with rates $\left(R_{1}, R_{2}\right)$ at first and second stages if there exists a sequence of $\left(M_{1}, M_{2}, d_{1}, d_{2}\right)$ average distortion codes for $\left(P_{X^{n}}, \mathrm{~d}_{1}^{(n)}, \mathrm{d}_{2}^{(n)}\right)$ with

$$
\begin{aligned}
& \limsup _{n \rightarrow \infty} \frac{1}{n} \log M_{1} \leq R_{1}, \\
& \limsup _{n \rightarrow \infty} \frac{1}{n} \log M_{2} \leq R_{2} .
\end{aligned}
$$

Rimoldi [16] showed that for the discrete memoryless source, the distortions $\left(d_{1}, d_{2}\right)$ are asymptotically attainable with rates $\left(R_{1}, R_{2}\right)$ at first and second stages if and only if

$$
\begin{array}{rl}
I\left(X ; Y_{1}\right) \leq R_{1} & \mathbb{E}\left[\mathrm{d}_{1}\left(X, Y_{1}\right)\right] \leq d_{1} \\
I\left(X ; Y_{1}, Y_{2}\right) \leq R_{2} & \mathbb{E}\left[\mathrm{d}_{2}\left(X, Y_{2}\right)\right] \leq d_{2},
\end{array}
$$

where here and in the sequel, $R_{2}$ refers to the total rate at both stages (see Effros [17] for a generalization to continuous alphabets and stationary sources). It is convenient to consider the following equivalent representation of the boundary of the set in (18):

$$
R_{2}\left(d_{1}, d_{2}, R_{1}\right) \triangleq \inf \left\{R_{2}:\left(d_{1}, d_{2}, R_{1}, R_{2}\right) \text { satisfy }(18)\right\} .
$$

Henceforth, we refer to the function $R_{2}\left(d_{1}, d_{2}, R_{1}\right): \mathbb{R}_{+}^{3} \mapsto$ $\mathbb{R}_{+}$as the second stage rate-distortion function. It represents 
the minimum asymptotically achievable total rate compatible with rate $R_{1}$ at the first stage and at-stage distortions $d_{1}, d_{2}$. For any achievable $\left(R_{1}, R_{2}, d_{1}, d_{2}\right)$, the following bound in terms of the standard rate-distortion function in (1) clearly holds:

$$
\begin{aligned}
& R_{1} \geq R_{\mathrm{d}_{1}}\left(d_{1}\right) \\
& R_{2} \geq R_{\mathrm{d}_{2}}\left(d_{2}\right)
\end{aligned}
$$

where $R_{\mathrm{d}_{1}}(\cdot)$ and $R_{\mathrm{d}_{2}}(\cdot)$ denote the rate-distortion functions for distortion measures $\mathrm{d}_{1}$ and $\mathrm{d}_{2}$, respectively. In Fig. 1, $\left(d_{1}, d_{2}\right)$ are fixed, and the region of achievable $\left(R_{1}, R_{2}\right)$ is greyed out; $R_{2}\left(d_{1}, d_{2}, R_{1}\right)$ is its boundary drawn in red. If the point $\left(R_{\mathrm{d}_{1}}\left(d_{1}\right), R_{\mathrm{d}_{2}}\left(d_{2}\right)\right)$ is attainable, the source is said to be successively refinable [18] at $\left(d_{1}, d_{2}\right)$.

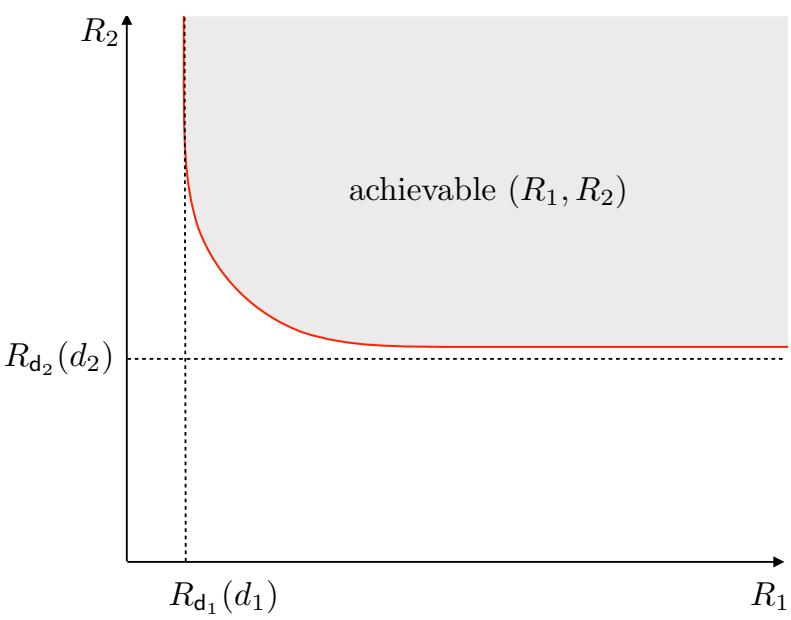

Fig. 1. The rate-distortion region for successive refinement, for fixed $d_{1}, d_{2}$ Note that if $\mathrm{d}_{1}=\mathrm{d}_{2}$, and $d_{2}<d_{1}$, then $R_{2}\left(d_{1}, d_{2}, R_{1}\right)=R\left(d_{2}\right)$ is attained at some $R_{1}<\infty$.

Throughout the paper, we assume that the following conditions are met.

(a) $R_{2}\left(d_{1}, d_{2}, R_{1}\right)$ is finite in some nonempty region $\subseteq \mathbb{R}_{+}^{3}$.

(b) There exist transition probability kernels $P_{Y_{1}^{\star} \mid X}$ and $P_{Y_{2}^{\star} \mid X Y_{1}^{\star}}$ that attain the infimum in (19).

The mild assumption (b) is always satisfied, for example, if $\mathcal{Y}_{1}, \mathcal{Y}_{2}$ are finite; if $\mathcal{X}$ is Polish, $\mathcal{Y}_{1}, \mathcal{Y}_{2}$ are compact metric, and distortion measures $\mathrm{d}_{1}, \mathrm{~d}_{2}$ are jointly continuous; and if $\mathcal{X}=\mathcal{Y}_{1}=\mathcal{Y}_{2}$ are Euclidean spaces with $\mathrm{d}_{1}(x, y) \rightarrow \infty$, $\mathrm{d}_{2}(x, y) \rightarrow \infty$ as $\|x-y\| \rightarrow \infty$ [2].

The second stage rate-distortion function $R_{2}\left(d_{1}, d_{2}, R_{1}\right)$ is nondecreasing and jointly convex in $\left(d_{1}, d_{2}, R_{1}\right)$ (see Lemma 3 in Section $\mathrm{V}$ below). The region of $\left(d_{1}, d_{2}, R_{1}\right)$ where the constraints are satisfied with equality is defined as follows.

$$
\begin{aligned}
\Omega \triangleq\{ & \left(d_{1}, d_{2}, R_{1}\right) \in \mathbb{R}_{+}^{3}: \forall\left(\epsilon_{1}, \epsilon_{2}, \epsilon_{3}\right)>0: \\
& \left.R_{2}\left(d_{1}+\epsilon_{1}, d_{2}+\epsilon_{2}, R_{1}+\epsilon_{3}\right)<R_{2}\left(d_{1}, d_{2}, R_{1}\right)<\infty\right\} .
\end{aligned}
$$

In the important special case of $\mathrm{d}_{1}=\mathrm{d}_{2}$,

$\Omega=\left\{\left(d_{1}, d_{2}, R_{1}\right): R\left(d_{1}\right)<R_{1}<R\left(d_{2}\right), d_{1} \leq d_{\max }\right\}$,

where $d_{\max }$ is the smallest positive scalar such that $R(d)$ is constant on $\left(d_{\max }, \infty\right)$.
Since $R_{2}\left(d_{1}, d_{2}, R_{1}\right)$ is convex in its input, each point $\left(d_{1}, d_{2}, R_{1}\right) \in \mathbb{R}_{+}^{3}$ on the curve can be parametrized via the supporting hyperplane $h-\lambda_{1}^{\star} d_{1}-\lambda_{2}^{\star} d_{2}-\nu_{1}^{\star} R_{1}=0$. Here $h$ is the is the distance of the hyperplane from the origin, and the triple $\left(\lambda_{1}^{\star}, \lambda_{2}^{\star}, \nu_{1}^{\star}\right)$ defines the normal vector to the hyperplane. Thus, to each $\left(d_{1}, d_{2}, R_{1}\right) \in \mathbb{R}_{+}^{3}$ there corresponds a triplet $\left(\lambda_{1}^{\star}, \lambda_{2}^{\star}, \nu_{1}^{\star}\right) \in \mathbb{R}_{+}^{3}$ such that for some $h \in \mathbb{R}_{+}$, the hyperplane $h-\lambda_{1}^{\star} d_{1}-\lambda_{2}^{\star} d_{2}-\nu_{1}^{\star} R_{1}=0$ is tangent to $R_{2}\left(d_{1}, d_{2}, R_{1}\right)$ at $\left(d_{1}, d_{2}, R_{1}\right)$.

Before we state our main result, we present the following notation. For measurable functions $\beta_{1}: \mathcal{X} \mapsto \mathbb{R}_{+}, \beta_{2}: \mathcal{X} \times$ $\mathcal{Y}_{1} \mapsto \mathbb{R}_{+}$and nonnegative numbers $\lambda_{1}, \lambda_{2}, \nu_{1}$, denote

$$
\begin{aligned}
\Sigma_{2}\left(y_{1}, y_{2}\right) & \triangleq \mathbb{E}\left[\frac{\exp \left(-\frac{\lambda_{1}}{1+\nu_{1}} \mathrm{~d}_{1}\left(X, y_{1}\right)-\lambda_{2} \mathrm{~d}_{2}\left(X, y_{2}\right)\right)}{\beta_{1}(X) \beta_{2}\left(X \mid y_{1}\right)^{\frac{\nu_{1}}{1+\nu_{1}}}}\right] \\
\Sigma_{1}\left(y_{1}\right) & \triangleq \mathbb{E}\left[\frac{\exp \left(-\frac{\lambda_{1}}{1+\nu_{1}} \mathrm{~d}_{1}\left(X, y_{1}\right)\right)}{\beta_{1}(X) \beta_{2}\left(X \mid y_{1}\right)^{-\frac{1}{1+\nu_{1}}}}\right]
\end{aligned}
$$

The quantities $\Sigma_{1}\left(y_{1}\right)$ and $\Sigma_{2}\left(y_{1}, y_{2}\right)$ generalize the expectation on the left side of (5) to successive refinement.

The main result of the paper can now be stated as follows.

Theorem 2 (Parametric representation). Assume that $\left(d_{1}, d_{2}, R_{1}\right) \in \Omega$. The boundary of the rate-distortion region of successive refinement can be represented as

$$
\begin{aligned}
& R_{2}\left(d_{1}, d_{2}, R_{1}\right) \\
= & \max \left\{\mathbb{E}\left[\log \frac{1}{\beta_{1}(X)^{1+\nu_{1}}}\right]-\lambda_{1} d_{1}-\lambda_{2} d_{2}-\nu_{1} R_{1}\right\},
\end{aligned}
$$

where the maximization is over $\left(\beta_{1}(x), \nu_{1}, \lambda_{1}, \lambda_{2}\right) \geq 0$ satisfying, for some $\beta_{2}\left(x \mid y_{1}\right) \geq 0$, the constraints

$$
\begin{aligned}
\Sigma_{2}\left(y_{1}, y_{2}\right) & \leq 1 \\
\Sigma_{1}\left(y_{1}\right) & \leq 1
\end{aligned}
$$

for all $\left(y_{1}, y_{2}\right) \in \mathcal{Y}_{1} \times \mathcal{Y}_{2}$.

Furthermore, in order for $\left(P_{Y_{1}^{*} \mid X}, P_{Y_{2}^{*} \mid X Y_{1}^{*}}\right)$ to achieve the infimum in (19), it is necessary and sufficient that

$$
\begin{aligned}
\frac{d P_{X \mid Y_{1}^{\star}=y_{1}}}{d P_{X}}(x) & =\frac{\exp \left(-\frac{\lambda_{1}^{\star}}{1+\nu_{1}^{\star}} \mathrm{d}_{1}\left(x, y_{1}\right)\right)}{\beta_{1}(x) \beta_{2}\left(x \mid y_{1}\right)^{-\frac{1}{1+\nu_{1}^{\star}}}}, \\
\frac{d P_{X \mid Y_{1}^{\star}=y_{1}, Y_{2}^{\star}=y_{2}}}{d P_{X \mid Y_{1}^{\star}=y_{1}}}(x) & =\frac{\exp \left(-\lambda_{2}^{\star} \mathrm{d}_{2}\left(x, y_{2}\right)\right)}{\beta_{2}\left(x \mid y_{1}\right)},
\end{aligned}
$$

where

$$
\left(\lambda_{1}^{\star}, \lambda_{2}^{\star}, \nu_{1}^{\star}\right)=-\nabla R_{2}\left(d_{1}, d_{2}, R_{1}\right)
$$

and $0 \leq \beta_{1}(x) \leq 1,0 \leq \beta_{2}\left(x \mid y_{1}\right) \leq 1$ satisfy

$$
\begin{aligned}
\Sigma_{2}\left(y_{1}, y_{2}\right) & \leq \Sigma_{1}\left(y_{1}\right) \\
& \leq 1
\end{aligned}
$$


for all $\left(y_{1}, y_{2}\right) \in \mathcal{Y}_{1} \times \mathcal{Y}_{2}$. Finally, the choice

$$
\begin{aligned}
\beta_{1}^{\star}(x) & =\mathbb{E}\left[\beta_{2}\left(x \mid Y_{1}^{\star}\right)^{\frac{1}{1+\nu_{1}^{\star}}} \exp \left(-\frac{\lambda_{1}^{\star}}{1+\nu_{1}^{\star}} \mathrm{d}_{1}\left(x, Y_{1}^{\star}\right)\right)\right], \\
\beta_{2}^{\star}\left(x \mid y_{1}\right) & =\mathbb{E}\left[\exp \left(-\lambda_{2}^{\star} \mathrm{d}_{2}\left(x, Y_{2}^{\star}\right)\right) \mid Y_{1}^{\star}=y_{1}\right] .
\end{aligned}
$$

satisfies (29), (30), (32), (33) and thus achieves the maximum in (26). Equality in (33) is attained for $P_{Y_{1}^{\star}-a . e .} y_{1}$, and equality in (32) is attained for $P_{Y_{2}^{\star} \mid Y_{1}^{\star}=y_{1}}$-a.e. $y_{2}{ }^{3}$

If the source is successively refinable at $\left(d_{1}, d_{2}\right)$, then the optimal choice is

$$
\begin{aligned}
\beta_{1}(x) & =\alpha_{1}^{\star}(x)^{\frac{\nu_{1}}{1+\nu_{1}}} \alpha_{2}^{\star}(x)^{\frac{1}{1+\nu_{1}}}, \\
\beta_{2}\left(x \mid y_{1}\right) & =\exp \left(-\frac{\lambda_{1}}{\nu_{1}} \mathrm{~d}_{1}\left(x, y_{1}\right)\right) \alpha_{1}^{\star-1}(x) \alpha_{2}^{\star}(x), \\
\lambda_{1} & =-\nu_{1} R_{\mathrm{d}_{1}}^{\prime}\left(d_{1}\right), \\
\lambda_{2} & =-R_{\mathrm{d}_{2}}^{\prime}\left(d_{2}\right) .
\end{aligned}
$$

for an arbitrary $\nu_{1}>0$, where $\alpha_{1}^{\star}(\cdot), \alpha_{2}^{\star}(\cdot)$ achieve the maximum of (4) for $\left\{\mathrm{d}_{1}, d_{1}\right\}$ and $\left\{\mathrm{d}_{2}, d_{2}\right\}$, respectively. It is easy to verify that in this case, (27) and (28) are satisfied, and the function in (26) equals $R_{\mathrm{d}_{2}}\left(d_{2}\right)$ when $R_{1}=R_{\mathrm{d}_{1}}\left(d_{1}\right)$. Plugging (36), (37) into (29), (30) yields the optimal kernels

$$
\begin{aligned}
d P_{X \mid Y_{1}^{\star}=y_{1}}(x) & =\frac{\exp \left(-\frac{\lambda_{1}}{\nu_{1}} \mathrm{~d}_{1}\left(x, y_{1}\right)\right)}{\alpha_{1}^{\star}(x)} d P_{X}(x), \\
d P_{X \mid Y_{1}^{\star}=y_{1}, Y_{2}^{\star}=y_{2}} & =\frac{\exp \left(-\lambda_{2} \mathrm{~d}_{2}\left(x, y_{2}\right)\right)}{\alpha_{2}^{\star}(x)} \frac{\alpha_{1}^{\star}(x) d P_{X \mid Y_{1}^{\star}=y_{1}}}{\exp \left(-\frac{\lambda_{1}}{\nu_{1}} \mathrm{~d}_{1}\left(x, y_{1}\right)\right)} \\
& =\frac{\exp \left(-\lambda_{2} \mathrm{~d}_{2}\left(x, y_{2}\right)\right)}{\alpha_{2}^{\star}(x)} d P_{X}(x),
\end{aligned}
$$

which coincide with the kernels that achieve the single-stage rate-distortion function (6), indicating successive refinability. The intuition is as follows. After the first stage of successive refinement is complete, the effective source distribution to be compressed is $P_{X \mid Y_{1}^{\star}}$. Due to (42), the Markov chain condition $P_{X \mid Y_{1}^{\star}, Y_{2}^{\star}}=P_{X \mid Y_{2}^{\star}}$ holds, where $P_{X \mid Y_{2}^{\star}}$ is the backward transition probability kernel that achieves the rate-distortion function at $d_{2}$ for $P_{X}$. Thus after the second stage the effective source distribution coincides with that of the optimal single-stage rate-distortion code, $P_{X \mid Y_{2}^{\star}}$. The calculation (42) also recovers the Markovian characterization of successive refinability due to Equitz and Cover [18, Th. 2].

Theorem 2 refines a prior finite alphabet result by Tuncel and Rose [3, Th. 4] and extends it to abstract probability spaces. In the finite alphabet case, the optimality conditions (34), (35) and (32), (33) were stated in [3, eq. (47), eq. (46) and eq. (50)], respectively. The dual representation of the rate-distortion region as a maximum over functions in (26) is new. One reason why such a representation is useful is that by choosing $\beta_{1}$ and $\beta_{2}$ appropriately, one can generate outer bounds to the rate-distortion region. For example, choosing $\beta_{1}$

\footnotetext{
${ }^{3}$ By the definition of a transition probability kernel, the transition probability kernels $P_{Y_{2}^{\star} \mid X, Y_{1}=y_{1}}$ and $P_{Y_{2}^{\star} \mid Y_{1}^{\star}=y_{1}}$ are well defined at every $y_{1}$ (and not only at $P_{\left.Y_{1}^{\star} \text {-a.e. } y_{1}\right)}^{2}$
}

and $\beta_{2}$ as in (36) and (37) leads to an outer bound to the ratedistortion region in (18), even if the source is not successively refinable. This particular choice also leads to a nonasymptotic converse bound in Corollary 1 in Section III below.

\section{NONASYMPTOTIC CONVERSE BOUND}

We focus on excess distortion codes for successive refinement, that we formally define as follows. An $\left(M_{1}, M_{2}, d_{1}, d_{2}, \epsilon_{1}, \epsilon_{2}\right)$ code for $\left(P_{X}, \mathrm{~d}_{1}, \mathrm{~d}_{2}\right)$ is a pair of encoders $\left(f_{1}, f_{2}\right)(10),(11)$ and decoders $\left(g_{1}, g_{2}\right)(12)$, (13) such that

$$
\begin{aligned}
& \mathbb{P}\left[\mathcal{A}_{1}^{c}\right] \leq \epsilon_{1}, \\
& \mathbb{P}\left[\mathcal{A}_{2}^{c}\right] \leq \epsilon_{2},
\end{aligned}
$$

where $\mathcal{A}_{1}$ and $\mathcal{A}_{2}$ denote the successful decoding events at first and second stages, respectively:

$$
\begin{aligned}
& \mathcal{A}_{1} \triangleq\left\{\mathrm{d}_{1}\left(X, Y_{1}\right) \leq d_{1}\right\}, \\
& \mathcal{A}_{2} \triangleq \mathcal{A}_{1} \cap\left\{\mathrm{d}_{2}\left(X, Y_{2}\right) \leq d_{2}\right\},
\end{aligned}
$$

where $Y_{1}=\mathrm{g}_{1}\left(\mathrm{f}_{1}(X)\right)$ and $\left.Y_{2}=\mathrm{g}_{2}\left(\mathrm{f}_{1}(X), \mathrm{f}_{2}(X)\right)\right)$. We allow randomized encoders and decoders, in which case $f_{1}, f_{2}, g_{1}, g_{2}$ are transition probability kernels rather than deterministic mappings.

It was shown in [11] that for single stage compression, the random variable called tilted information, defined in (9), plays the key role in the corresponding nonasymptotic fundamental limits. Leveraging the result of Theorem 2, we can define the tilted information for successive refinement as follows.

Definition 1. Fix $P_{X}, \mathrm{~d}_{1}, \mathrm{~d}_{2}$. Tilted information for successive refinement of $x$ at $\left(d_{1}, d_{2}, R_{1}\right) \in \Omega$ is defined as

$\jmath\left(x, d_{1}, d_{2}, R_{1}\right) \triangleq\left(1+\nu_{1}^{\star}\right) \log \frac{1}{\beta_{1}^{\star}(x)}-\lambda_{1}^{\star} d_{1}-\lambda_{2}^{\star} d_{2}-\nu_{1}^{\star} R_{1}$

where $\left(\beta_{1}^{\star}(\cdot), \lambda_{1}^{\star}, \lambda_{2}^{\star}, \nu_{1}^{\star}\right)$ achieve the maximum in (26).

If the source is successively refinable at $d_{1}, d_{2}$, then the tilted information for successive refinement coincides with the tilted information for single stage compression:

$$
\jmath\left(x, d_{1}, d_{2}, R_{\mathrm{d}_{1}}\left(d_{1}\right)\right)=\jmath_{\mathrm{d}_{2}}\left(x, d_{2}\right) .
$$

Fixing $\left(\beta_{1}(\cdot), \lambda_{1}, \lambda_{2}, \nu_{1}\right) \geq 0$ that satisfy (27) and (28), for some $\beta_{2}(\cdot \mid \cdot) \geq 0$, the notion of tilted information can be generalized by defining

$$
F \triangleq\left(1+\nu_{1}\right) \log \frac{1}{\beta_{1}(x)}-\lambda_{1} d_{1}-\lambda_{2} d_{2}-\nu_{1} \log M_{1} .
$$

Choosing $\left(\beta_{1}(\cdot), \lambda_{1}, \lambda_{2}, \nu_{1}\right)=\left(\beta_{1}^{\star}(\cdot), \lambda_{1}^{\star}, \lambda_{2}^{\star}, \nu_{1}^{\star}\right)$ as in Definition 1 would result in $F=\jmath\left(X, d_{1}, d_{2}, \log M_{1}\right)$. For a given $\left(M_{1}, M_{2}, d_{1}, d_{2}, \epsilon_{1}, \epsilon_{2}\right)$ code $\left(\mathrm{f}_{1}, \mathrm{f}_{2}, \mathrm{~g}_{1}, \mathrm{~g}_{2}\right)$ with $Y_{1}=$ $\mathrm{g}_{1}\left(\mathrm{f}_{1}(X)\right)$, it is instructive to split (49) into two terms (corresponding to both stages of successive refinement):

$$
F=\nu_{1}\left(F_{1}-\log M_{1}\right)+F_{2},
$$


where

$$
\begin{aligned}
& F_{1} \triangleq \log \frac{\beta_{2}\left(X \mid Y_{1}\right)^{\frac{1}{1+\nu_{1}}}}{\beta_{1}(X)}-\frac{\lambda_{1}}{1+\nu_{1}} d_{1}, \\
& F_{2} \triangleq \log \frac{\beta_{2}\left(X \mid Y_{1}\right)^{-\frac{\nu_{1}}{1+\nu_{1}}}}{\beta_{1}(X)}-\frac{\lambda_{1}}{1+\nu_{1}} d_{1}-\lambda_{2} d_{2} .
\end{aligned}
$$

Roughly speaking, $F_{1}$ and $F_{2}$ represent the estimates of the number of bits about $X$ that need be conveyed at the end of first and second stages in order to satisfy the constraints $\mathrm{d}_{1}\left(X, Y_{1}\right) \leq d_{1}$ and $\mathrm{d}_{1}\left(X, Y_{2}\right) \leq d_{2}$, respectively, i.e. the information content of $X$ relevant to satisfying these constraints. Since we are looking at a fixed rate scenario, and $F_{1}$ and $F_{2}$ are random variables, we expect the excess distortion event to occur once the information contents $F_{1}$ and $F_{2}$ exceed those chosen fixed rates. This intuition is made rigorous in the next result, which states that the probability that $F_{1}, F_{2}$ are too high for the chosen rates yet the decoding is performed correctly is low.

Theorem 3. For an $\left(M_{1}, M_{2}, d_{1}, d_{2}, \epsilon_{1}, \epsilon_{2}\right)$ code to exist, it is necessary that for all $\left(\gamma_{1}, \gamma_{2}\right)>0$,

$$
\begin{aligned}
& \mathbb{P}\left[\left\{F_{1} \geq \log M_{1}+\gamma_{1}\right\} \cap \mathcal{A}_{1}\right] \leq \exp \left(-\gamma_{1}\right), \\
& \mathbb{P}\left[\left\{F_{2} \geq \log M_{2}+\gamma_{2}\right\} \cap \mathcal{A}_{2}\right] \leq \exp \left(-\gamma_{2}\right),
\end{aligned}
$$

where $\mathcal{A}_{1}, \mathcal{A}_{2}$ are the successful decoding events (45), (46).

Proof of Theorem 3. We employ Theorem 2 similar to how Theorem 1 was employed in the proof of [11, Th. 7].

Let the two-stage encoder and decoder be the random transformations $\left(P_{W_{1} \mid X}, P_{W_{2} \mid X, W_{1}}\right)$ and $\left(P_{Y_{1} \mid W_{1}}, P_{Y_{2} \mid W_{1}, W_{2}}\right)$, where $W_{1}$ takes values in $\left\{1, \ldots, M_{1}\right\}$, and $W_{2}$ takes values in $\left\{1, \ldots,\left\lfloor M_{2} / M_{1}\right\rfloor\right\}$.

Furthermore, introduce the auxiliary distribution $Q_{W_{1} W_{2}}$, equiprobable on $\left\{1, \ldots, M_{1}\right\} \times\left\{1, \ldots,\left\lfloor M_{2} / M_{1}\right\rfloor\right\}$, and let $Q_{Y_{1} Y_{2}}$ be the distribution on $\mathcal{Y}_{1} \times \mathcal{Y}_{2}$ that arises after $Q_{W_{1} W_{2}}$ is passed through the random transformation $P_{Y_{1} Y_{2} \mid W_{1}, W_{2}}$ defined by our code, i.e. $Q_{W_{1} W_{2}} \rightarrow P_{Y_{1} Y_{2} \mid W_{1}, W_{2}} \rightarrow Q_{Y_{1} Y_{2}}$.

To show (65), write, for any $\gamma_{1} \geq 0$

$$
\begin{aligned}
& \mathbb{P}\left[\left\{F_{1} \geq \log M_{1}+\gamma_{1}\right\} \cap \mathcal{A}_{1}\right] \\
\leq & \int_{x \in \mathcal{X}} d P_{X}(x) \sum_{w=1}^{M_{1}} P_{W_{1} \mid X=x}(w) \int d P_{Y_{1} \mid W_{1}=w}(y) \\
& \cdot 1\left\{M_{1} \leq \exp \left(F_{1}-\gamma_{1}\right)\right\} 1\left\{\mathrm{~d}_{1}\left(x, y_{1}\right) \leq d_{1}\right\} \\
\leq & \exp \left(-\gamma_{1}\right) \mathbb{E}_{P_{X} \times Q_{Y_{1}}}\left[\exp \left(F_{1}\right) 1\left\{\mathrm{~d}_{1}\left(X, Y_{1}\right) \leq d_{1}\right\}\right] \\
\leq & \exp \left(-\gamma_{1}\right) \mathbb{E}_{Q_{Y_{1}}}\left[\Sigma_{1}\left(Y_{1}\right)\right] \\
\leq & \exp \left(-\gamma_{1}\right)
\end{aligned}
$$

where

- (56) follows by upper-bounding $P_{W_{1} \mid X=x}(w) \leq 1$, and

$$
1\left\{M_{1} \leq \exp \left(F_{1}-\gamma_{1}\right)\right\} \leq \frac{\exp \left(-\gamma_{1}\right)}{M_{1}} \exp \left(F_{1}\right) ;
$$

- (58) is due to (28).
We proceed to show (66). We have, for any $\gamma_{2} \geq 0$

$$
\begin{aligned}
& \mathbb{P}\left[\left\{F_{2} \geq \log M_{2}+\gamma_{2}\right\} \cap \mathcal{A}_{2}\right] \\
\leq & \int_{x \in \mathcal{X}} d P_{X}(x) \sum_{w_{1}, w_{2}} P_{W_{1}, W_{2} \mid X=x}\left(w_{1}, w_{2}\right) \\
& \cdot \mathbb{E}\left[1\left\{M_{2} \leq \exp \left(F_{2}-\gamma_{2}\right)\right\}\right. \\
& \left.\quad \cdot 1\left\{\mathcal{A}_{2}\right\} \mid X=x, W_{1}=w_{1}, W_{2}=w_{2}\right] \\
\leq & \exp \left(-\gamma_{2}\right) \mathbb{E}_{P_{X} \times Q_{Y_{1} Y_{2}}\left[\exp \left(F_{2}\right) 1\left\{\mathcal{A}_{2}\right\}\right]} \\
\leq & \exp \left(-\gamma_{2}\right) \mathbb{E}_{Q_{Y_{1} Y_{2}}}\left[\Sigma_{2}\left(Y_{1}, Y_{2}\right)\right] \\
\leq & \exp \left(-\gamma_{2}\right),
\end{aligned}
$$

where

- (61) follows by upper-bounding $P_{W_{1}, W_{2} \mid X=x}\left(w_{1}, w_{2}\right) \leq$ 1 , and

$$
1\left\{M_{2} \leq \exp \left(F_{2}-\gamma_{2}\right)\right\} \leq \frac{\exp \left(-\gamma_{2}\right)}{M_{2}} \exp \left(F_{2}\right) ;
$$

- (63) is due to (27).

Theorem 3 immediately leads to the following converse: for an $\left(M_{1}, M_{2}, d_{1}, d_{2}, \epsilon_{1}, \epsilon_{2}\right)$ code to exist, it is necessary that for all $\left(\gamma_{1}, \gamma_{2}\right)>0$,

$$
\begin{aligned}
& \epsilon_{1} \geq \mathbb{P}\left[F_{1} \geq \log M_{1}+\gamma_{1}\right]-\exp \left(-\gamma_{1}\right), \\
& \epsilon_{2} \geq \mathbb{P}\left[F_{2} \geq \log M_{2}+\gamma_{2}\right]-\exp \left(-\gamma_{2}\right) .
\end{aligned}
$$

In general, $F_{1}$ and $F_{2}$ are functions of a given code, which limits the computability of the basic converse in Theorem 3 or that in (65), (66). Fortunately, via elementary probability rules, Theorem 3 immediately leads to a series of corollaries that are computable and useful in several applications as explained below.

The following corollary to Theorem 3 is immediate from the observation that (36)-(39) satisfy (27) and (28), and thus $F_{1}=\jmath_{\mathrm{d}_{1}}\left(X, d_{1}\right)$ and $F_{2}=\jmath_{\mathrm{d}_{2}}\left(X, d_{2}\right)$ is a valid choice for these functions.

Corollary 1. Fix an $\left(M_{1}, M_{2}, d_{1}, d_{2}, \epsilon_{1}, \epsilon_{2}\right)$ code. Then, for all $\left(\gamma_{1}, \gamma_{2}\right)>0$, it holds that

$$
\begin{aligned}
& \epsilon_{1} \geq \mathbb{P}\left[{\jmath \mathrm{d}_{1}}_{1}\left(X, d_{1}\right) \geq \log M_{1}+\gamma_{1}\right]-\exp \left(-\gamma_{1}\right) \\
& \epsilon_{2} \geq \mathbb{P}\left[\jmath_{\mathrm{d}_{2}}\left(X, d_{2}\right) \geq \log M_{2}+\gamma_{2}\right]-\exp \left(-\gamma_{2}\right) .
\end{aligned}
$$

where $\jmath_{\mathrm{d}_{1}}$ and $\jmath_{\mathrm{d}_{2}}$ are the $\mathrm{d}_{1}$ - and $\mathrm{d}_{2}$-tilted informations (defined in (9)), respectively.

Corollary 1 applies whether or not the source is successively refinable.

The next corollary recombines the $F_{1}$ and $F_{2}$ events in Theorem 3 to yield a bound on the joint error probability $\epsilon_{2}$ in terms of $F$ and $F_{1}$. This is useful when $F_{1}$ is a function of $X$ only; for example when $F_{1}=\jmath_{\mathrm{d}_{1}}\left(X, d_{1}\right)$.

Corollary 2. Fix an $\left(M_{1}, M_{2}, d_{1}, d_{2}, \epsilon_{1}, \epsilon_{2}\right)$ code. For all $\left(\gamma_{1}, \gamma_{2}\right)>0$, both (65) and

$$
\begin{aligned}
\epsilon_{2} \geq & \mathbb{P} \\
& {\left[\left\{F \geq \log M_{2}+\nu_{1} \gamma_{1}+\gamma_{2}\right\} \cup\left\{F_{1} \geq \log M_{1}+\gamma_{1}\right\}\right] } \\
& -\exp \left(-\gamma_{1}\right)-\exp \left(-\gamma_{2}\right)
\end{aligned}
$$




\section{must hold.}

Proof. Consider the event

$$
\mathcal{B} \triangleq\left\{F \geq \log M_{2}+\nu_{1} \gamma_{1}+\gamma_{2}\right\} \cup\left\{F_{1} \geq \log M_{1}+\gamma_{1}\right\} .
$$

Using elementary probability laws and Theorem 3, write

$$
\begin{aligned}
\mathbb{P}[\mathcal{B}]= & \mathbb{P}\left[\mathcal{B} \cap \mathcal{A}_{2}^{c}\right]+\mathbb{P}\left[\mathcal{B} \cap \mathcal{A}_{2}\right] \\
\leq & \epsilon+\mathbb{P}\left[\mathcal{B} \cap \mathcal{A}_{2} \cap\left\{F_{1} \geq \log M_{1}+\gamma_{1}\right\}\right] \\
& +\mathbb{P}\left[\mathcal{B} \cap \mathcal{A}_{2} \cap\left\{F_{1}<\log M_{1}+\gamma_{1}\right\}\right] \\
\leq & \epsilon+\exp \left(-\gamma_{1}\right)+\mathbb{P}\left[\mathcal{A}_{2} \cap\left\{F_{2} \geq \log M_{2}+\gamma_{2}\right\}\right] \\
\leq & \epsilon+\exp \left(-\gamma_{1}\right)+\exp \left(-\gamma_{2}\right)
\end{aligned}
$$

In general, $F_{1}$ is a function of a given code, which limits the computability of the converse in Corollary 2. However, when operating at first stage rate close to $R_{\mathrm{d}_{1}}\left(d_{1}\right)$, which corresponds to the vertical asymptote in Fig. $1, F_{1}$ becomes a function of $X$ only, and (69) gives a computable bound that is tighter than (75). Indeed, letting $\left(\beta_{1}(\cdot), \lambda_{1}, \lambda_{2}, \nu_{1}\right)$ to achieve the maximum in (26) at $\left(d_{1}, d_{2}, R_{\mathrm{d}_{1}}\left(d_{1}\right)\right)$, we obtain $F_{1}=\jmath_{\mathrm{d}_{1}}\left(X, d_{1}\right)$, which is a function of $X$ only, and $F=\jmath\left(X, d_{1}, d_{2}, R_{\mathrm{d}_{1}}\left(d_{1}\right)\right)+\nu_{1} R_{\mathrm{d}_{1}}\left(d_{1}\right)-\nu_{1} \log M_{1}$.

Omitting the $F_{1}$ event from the probability in (69) and choosing $\left(\beta_{1}(\cdot), \lambda_{1}, \lambda_{2}, \nu_{1}\right)$ as in Definition 1 so that $F=$ $\jmath\left(X, d_{1}, d_{2}, \log M_{1}\right)$, we obtain a bound on the joint error probability $\epsilon_{2}$ in terms of tilted information only, stated in Corollary 3 below. This is nice because it generalizes the corresponding result for one stage compression [11, Th. 7], and because it leads to a tight second-order result, as explained at the end of this section.

Corollary 3. For any $\left(M_{1}, M_{2}, d_{1}, d_{2}, \epsilon_{1}, \epsilon_{2}\right)$ code and for all $\left(\gamma_{1}, \gamma_{2}\right)>0$, it holds that

$$
\begin{gathered}
\epsilon_{2} \geq \mathbb{P}\left[\jmath\left(X, d_{1}, d_{2}, \log M_{1}\right) \geq \log M_{2}+\nu_{1}^{\star} \gamma_{1}+\gamma_{2}\right] \\
-\exp \left(-\gamma_{1}\right)-\exp \left(-\gamma_{2}\right)
\end{gathered}
$$

In a typical application of the bound in Corollary $3, \gamma_{1}$ and $\gamma_{2}$ will be chosen so that the terms $\nu_{1}^{\star} \gamma_{1}+\gamma_{2}$ inside the probability and $\exp \left(-\gamma_{1}\right)+\exp \left(-\gamma_{2}\right)$ outside are both negligible. Thus, Corollary 3 establishes that the excess-distortion probability is roughly bounded below by the complementary cdf of tilted information.

For successively refinable finite alphabet sources, No et al. [12] found the dispersion of successive refinement. The dispersion of non-successively refinable finite alphabet sources was recently computed in [13]. A straightforward second-order analysis (along the lines of [11, (103)-(106)]) of the bound in Corollary 1 recovers the converse parts of the dispersion results in [12] and [13], respectively, and extends them to abstract stationary memoryless sources. Specifically, let $Q^{-1}(\epsilon)$ be the inverse of the standard Gaussian complementary cdf and let $\mathbf{Q}^{-1}(\epsilon, \boldsymbol{\Sigma})$ be the $K$-dimensional analogue of that function for a Gaussian random vector with zero mean and covariance matrix $\boldsymbol{\Sigma}$, i.e. $\mathbf{Q}^{-1}(\epsilon, \boldsymbol{\Sigma})$ is the boundary of the set

$$
\left\{z \in \mathbb{R}^{K}: \mathbb{P}[\mathcal{N}(\mathbf{0}, \boldsymbol{\Sigma}) \leq z] \geq 1-\epsilon\right\} .
$$

Consider some $\left(R_{1}^{\star}, R_{2}^{\star}\right)$ on the boundary of the set in (18) and some $\left(L_{1}^{\star}, L_{2}^{\star}\right)$ on the boundary of the set

$$
\begin{aligned}
& \left\{\left(L_{1}, L_{2}\right) \in \mathbb{R}^{2}:\right. \\
& \left.\nu_{1}^{\star} L_{1}+L_{2} \leq \sqrt{\operatorname{Var}\left[\jmath\left(X, d_{1}, d_{2}, R_{1}^{\star}\right)\right]} Q^{-1}\left(\epsilon_{2}\right)\right\},
\end{aligned}
$$

where $\nu_{1}^{\star}$ is the negative of the derivative of $R_{2}\left(d_{1}, d_{2}, R_{1}\right)$ with respect to $R_{1}$ at $R_{1}=R_{1}^{\star}$. An asymptotic analysis of Corollary 3 yields an extension of the converse part of [13, Th. 11 (i)] to abstract alphabets: if an $\left(M_{1}, M_{2}, d_{1}, d_{2}, \epsilon_{1}, \epsilon_{2}\right)$ code exists for $n$ i.i.d. copies of $X$, then

$$
\begin{aligned}
& \log M_{1} \geq n R_{1}^{\star}+\sqrt{n} L_{1}^{\star}+O(\log n), \\
& \log M_{2} \geq n R_{2}^{\star}+\sqrt{n} L_{2}^{\star}+O(\log n) .
\end{aligned}
$$

When the asymptotic rate at first stage is the vertical asymptote in Fig. 1, i.e. $R_{1}^{\star}=R_{\mathrm{d}_{1}}\left(d_{1}\right)$, then Corollary 2 leads to the following strengthening of (78), (79): if an $\left(M_{1}, M_{2}, d_{1}, d_{2}, \epsilon_{1}, \epsilon_{2}\right)$ code exists for $n$ i.i.d. copies of $X$, then (78), (79) hold with $\left(L_{1}^{\star}, \nu^{\star} L_{1}^{\star}+L_{2}^{\star}\right) \in \mathbf{Q}^{-1}\left(\epsilon_{2}, \boldsymbol{\Sigma}\right)$ for $\boldsymbol{\Sigma}$ being the covariance matrix of the two-dimensional random vector $\left(\jmath_{\mathrm{d}_{1}}\left(X, d_{1}\right), \jmath\left(X, d_{1}, d_{2}, R_{1}^{\star}\right)\right)$. The finite alphabet case of this result is the converse part of [13, Th. 11 (iii)]. The converse result (78), (79) also holds with $R_{1}^{\star}=R_{\mathrm{d}_{1}}\left(d_{1}\right)$, $R_{2}^{\star}=R_{\mathrm{d}_{2}}\left(d_{2}\right)$, and $\boldsymbol{\Sigma}$ the covariance matrix of the twodimensional random vector $\left(\jmath_{\mathrm{d}_{1}}\left(X, d_{1}\right), \jmath_{\mathrm{d}_{2}}\left(X, d_{1}\right)\right)$, which is tight if the source is successively refinable [13, Cor. 13 (iii)].

Unlike [13] who focused on the joint probability of error $\epsilon_{2}$ without placing any further constraint on $\epsilon_{1}$ apart from the trivial $\epsilon_{1} \leq \epsilon_{2}$, No et al. [12] considered a formulation that places separate upper bounds on each of the probabilities that the source is not reproduced within distortion levels $d_{1}$ and $d_{2}$, i.e. (43) and $\mathbb{P}\left[\mathrm{d}_{2}\left(X, Y_{2}\right)>d_{2}\right] \leq \epsilon_{2}^{\prime}$. It is easy to show that Corollary 1 continues to hold with $\epsilon_{2}$ replaced by $\epsilon_{2}^{\prime}$. The converse part of [12, Cor. 6] then extend it to abstract alphabets as follows: if an $\left(M_{1}, M_{2}, d_{1}, d_{2}, \epsilon_{1}, \epsilon_{2}^{\prime}\right)$ code under separate error probability formalism exists for $n$ i.i.d. copies of $X$, then

$$
\begin{aligned}
\log M_{1} & \geq n R_{\mathrm{d}_{1}}\left(d_{1}\right)+\sqrt{n \operatorname{Var}\left[\jmath_{\mathrm{d}_{1}}\left(X, d_{1}\right)\right]} Q^{-1}\left(\epsilon_{1}\right) \\
& +O(\log n), \\
\log M_{2} & \geq n R_{\mathrm{d}_{2}}\left(d_{2}\right)+\sqrt{n \operatorname{Var}\left[\jmath_{\mathrm{d}_{2}}\left(X, d_{2}\right)\right]} Q^{-1}\left(\epsilon_{2}^{\prime}\right) \\
& +O(\log n) .
\end{aligned}
$$

\section{Proof of Theorem 1}

In this section, we revisit the beautiful proof of Theorem 1 by Csiszár [2]. We streamline Csiszár's argument by using the the Donsker-Varadhan characterization of the minimum relative entropy, stated below, which will be also instrumental in the proof of Theorem 2.

Lemma 1 (Donsker-Varadhan, [19, Lemma 2.1], [20, Th. 3.5] ). Let $\rho: \mathcal{X} \mapsto[-\infty,+\infty]$ and let $\bar{X}$ be a random variable on $\mathcal{X}$ such that $\mathbb{E}[\exp (-\rho(\bar{X}))]<\infty$. Then,

$$
D(X \| \bar{X})+\mathbb{E}[\rho(X)] \geq \log \frac{1}{\mathbb{E}[\exp (-\rho(\bar{X}))]}
$$


with equality if and only if $X$ has distribution $P_{X \star}$ such that

$$
d P_{X^{\star}}(x)=\frac{\exp (-\rho(x))}{\mathbb{E}[\exp (-\rho(\bar{X}))]} d P_{\bar{X}}(x)
$$

We now recall some useful general properties of $R(d)$.

Fix source distribution $P_{X}$. For some transition probability kernel $P=P_{Y \mid X}$, put

$$
\begin{aligned}
& I(P) \triangleq I(X ; Y) \\
& \rho(P) \triangleq \mathbb{E}[\mathrm{d}(X, Y)] .
\end{aligned}
$$

Lemma 2 ( [2, Lemma 1.1]). $R(d)$ is non-increasing, convex and

$$
R(d)=\inf _{P: \rho(P)=d} I(P) \quad d_{\min }<d \leq d_{\max } .
$$

Let $F(\lambda)$ denote the maximum of the vertical axis intercepts of the straight lines of slope $-\lambda$ which have no point above the $R(d)$ curve, i.e. using (86) for $\lambda>0$ (see Fig. 2) ${ }^{4}$

$$
F(\lambda) \triangleq \inf _{P} I(P)+\lambda \rho(P) .
$$

Furthermore, since $R(d)$ is convex and nonincreasing, to each

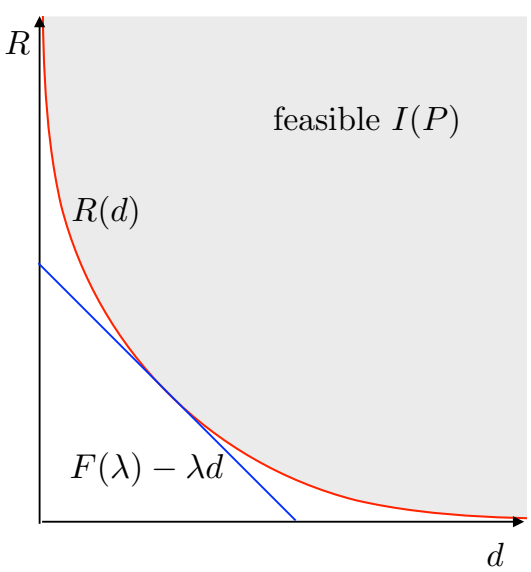

Fig. 2. Lagrange duality for the rate-distortion problem.

$d \geq d_{\text {min }}$, there exists $\lambda \geq 0$ such that the straight line of slope $-\lambda$ through $(d, R(d))$ is tangent to the $R(d)$ curve, and

$$
R(d) \triangleq \max _{\lambda \geq 0}(F(\lambda)-\lambda d) .
$$

Theorem 1 will follow from (88) and Theorem 4 below.

Theorem 4 (Necessary and sufficient conditions for an optimizer [2]). In order for $P_{Y^{*} \mid X}$ to achieve the infimum in (87), it is necessary and sufficient that

$$
\frac{d P_{X \mid Y^{\star}=y}}{d P_{X}}(x)=\frac{\exp (-\lambda \mathrm{d}(x, y))}{\alpha(x)}
$$

where $0 \leq \alpha(x) \leq 1$ satisfies (5). Furthermore, the choice

$$
\alpha^{*}(x)=\mathbb{E}\left[\exp \left(-\lambda \mathrm{d}\left(x, Y^{*}\right)\right)\right]
$$

\footnotetext{
${ }^{4}$ The optimization problem in (87) is known as the Lagrangian dual problem, and the function $F(\lambda)$ as the Lagrange dual.
}

satisfies (89) and (5), and for any $\alpha(x) \geq 0$ satisfying (5) we have for all $\tilde{P}$

$$
I(\tilde{P})+\lambda \rho(\tilde{P}) \geq \mathbb{E}\left[\log \frac{1}{\alpha(X)}\right]
$$

with equality if and only if $\tilde{P}$ can be represented as in (89), with the given $\alpha(x)$.

Proof of Theorem 4. Consider the function

$$
\begin{aligned}
L\left(P_{Y \mid X}, P_{\bar{Y}}\right) & =D\left(P_{Y \mid X} \| P_{\bar{Y}} \mid P_{X}\right)+\lambda \mathbb{E}[\mathrm{d}(X, Y)] \\
& =I(X ; Y)+D(Y \| \bar{Y})+\lambda \mathbb{E}[\mathrm{d}(X, Y)] \\
& \geq I(X, Y)+\lambda \mathbb{E}[\mathrm{d}(X, Y)]
\end{aligned}
$$

Since equality in (94) holds if and only if $P_{Y}=P_{\bar{Y}}, F(\lambda)$ can be expressed as

$$
F(\lambda)=\inf _{P_{\bar{Y}}} \inf _{P_{Y \mid X}} L\left(P_{Y \mid X}, P_{\bar{Y}}\right) .
$$

Denote

$$
\Sigma_{\bar{Y}}(x) \triangleq \mathbb{E}[\exp (-\lambda \mathrm{d}(x, \bar{Y}))] .
$$

Since $\mathrm{d}(x, y) \geq 0$, we have $0 \leq \Sigma_{\bar{Y}}(x) \leq 1$, and Lemma 1 applies to conclude that equality in

$$
D\left(P_{Y \mid X=x} \| P_{\bar{Y}}\right)+\lambda \mathbb{E}[\mathrm{d}(x, Y) \mid X=x] \geq \log \frac{1}{\Sigma_{\bar{Y}}(x)},
$$

is achieved if and only if $P_{Y \mid X=x}=P_{\bar{Y}^{*} \mid X=x}$, where $P_{\bar{Y}^{*} \mid X=x}$ is determined from

$$
\log \frac{d P_{\bar{Y}^{*} \mid X=x}(y)}{d P_{\bar{Y}}(y)}+\lambda \mathrm{d}(x, y)=\log \frac{1}{\Sigma_{\bar{Y}}(x)} .
$$

Applying (97) to solve for the inner minimizer in (95), we obtain

$$
\begin{aligned}
F(\lambda) & =\inf _{P_{\bar{Y}}} \mathbb{E}\left[\log \frac{1}{\Sigma_{\bar{Y}}(X)}\right] \\
& =\mathbb{E}\left[\log \frac{1}{\Sigma_{Y^{*}}(X)}\right]
\end{aligned}
$$

where (100) holds by the assumption (B).

Although for a fixed $P_{\bar{Y}}$ we can always define the tilted distribution $P_{\bar{Y}^{*} \mid X}$ via (98), in general we cannot claim that the marginal distribution $P_{\bar{Y}}$ that results after applying the random transformation $P_{\bar{Y}^{*} \mid X}$ to $P_{X}$ coincides with $P_{\bar{Y}}$. This happens if and only if $P_{\bar{Y}}$ is such that for $P_{\bar{Y}}$-a.e. $y$,

$$
\mathbb{E}\left[\frac{\exp (-\lambda \mathrm{d}(X, y))}{\Sigma_{\bar{Y}}(X)}\right]=1 \text {. }
$$

Since by the assumption (B), these exists $P_{Y^{*} \mid X}$ that achieves (100), condition (101) must hold for $P_{\bar{Y}}=P_{Y^{*}}$. Using this observation together with (100), we conclude that $P_{Y^{*} \mid X}$ in (89) with $\alpha(x)=\alpha^{*}(x)$, where $\alpha^{*}(x)$ is defined in (90), is necessary and sufficient to achieve the minimum of $F(\lambda)$ in (87). In particular, (89) is a necessary condition for the minimizer.

We now show that $\alpha^{*}(x)$ satisfies (5), which implies that both (89) and (5) are necessary. Since $P_{X} \rightarrow P_{Y^{*} \mid X} \rightarrow P_{Y^{*}}$, equality in (101) particularized to $P_{Y^{*}}$ holds for $P_{Y^{*}-\text { a.s. }} y$, 
which is equivalent to equality in (5). To show (5) for all $y$, note using (99) that for any $P_{\bar{Y}}$,

$$
\mathbb{E}\left[\log \frac{1}{\Sigma_{Y^{*}}(X)}\right] \leq \mathbb{E}\left[\log \frac{1}{\Sigma_{\bar{Y}}(X)}\right]
$$

For an arbitrary $\bar{y} \in \mathcal{Y}$ and $0 \leq \epsilon \leq 1$, let

$$
P_{\bar{Y}}=(1-\epsilon) P_{Y^{*}}+\epsilon \delta_{\bar{y}}
$$

for which

$$
\Sigma_{\bar{Y}}(x)=(1-\epsilon) \Sigma_{Y^{*}}(x)+\epsilon \exp \left(-\lambda^{*} \mathrm{~d}(x, \bar{y})\right)
$$

Substituting (104) in (102), we obtain

$$
\begin{aligned}
0 & \geq \mathbb{E}\left[\log \frac{\Sigma_{\bar{Y}}(X)}{\Sigma_{Y^{*}}(X)}\right] \\
& =\mathbb{E}\left[\log \left[1-\epsilon+\epsilon \frac{\exp (-\lambda \mathrm{d}(X, \bar{y}))}{\Sigma_{Y^{*}}(X)}\right]\right] \\
& =\log (1-\epsilon)+\mathbb{E}\left[\log \left[1+\frac{\epsilon}{1-\epsilon} \frac{\exp (-\lambda \mathrm{d}(X, \bar{y}))}{\Sigma_{Y^{*}}(X)}\right]\right]
\end{aligned}
$$

Since the difference quotient of the second term satisfies $0 \leq$ $\frac{1}{\epsilon} \log \left(1+\frac{\epsilon x}{1-\epsilon}\right) \leq \frac{x \log e}{1-\delta}$ for all $x \geq 0$ and $0 \leq \epsilon \leq \delta \leq 1$, by the dominated convergence theorem, the right derivative of (107) with respect to $\epsilon$ evaluated at $\epsilon=0$ is

$$
\mathbb{E}\left[-1+\frac{\exp (-\lambda \mathrm{d}(X, \bar{y}))}{\Sigma_{Y^{*}}(X)}\right] \log e \leq 0
$$

where the inequality holds because otherwise (105) would be violated for sufficiently small $\epsilon$. This concludes the proof that $\alpha^{*}(x)$ in (90) satisfies condition (5), so both (89) and (5) in Theorem 4 are necessary.

The sufficiency of (89) and (5) for $P_{Y^{*} \mid X}$ to achieve the minimum in (87) follows from (91). To show (91), fix any $\alpha(x)$ satisfying (5) and use the concavity of the logarithm to show that

$$
\begin{aligned}
& I(\tilde{P})+\lambda \rho(\tilde{P}) \\
\geq & \mathbb{E}\left[\log \frac{1}{\Sigma_{Y^{*}}(X)}\right] \\
= & \mathbb{E}\left[\log \frac{1}{\alpha(X)}\right]-\mathbb{E}\left[\log \frac{\Sigma_{Y^{*}}(X)}{\alpha(X)}\right] \\
\geq & \mathbb{E}\left[\log \frac{1}{\alpha(X)}\right]-\log \mathbb{E}_{P_{X} \times P_{Y^{*}}} \frac{\exp \left(-\lambda \mathrm{d}\left(X, Y^{*}\right)\right)}{\alpha(X)} \\
\geq & \mathbb{E}\left[\log \frac{1}{\alpha(X)}\right] .
\end{aligned}
$$

For the equality condition, observe that strict concavity of logarithm implies that equality in (111) holds if and only if the ratio $\frac{\Sigma_{Y^{*}}(X)}{\alpha(X)}$ is constant, while equality in (112) holds if and only if that constant is 1 .

\section{Proof of Theorem 2}

Fix the source distribution $P_{X}$. For a transition probability kernel $P=P_{Y_{1} Y_{2} \mid X}$, put

$$
\begin{aligned}
& I_{1}(P) \triangleq I\left(X ; Y_{1}\right) \\
& I_{2}(P) \triangleq I\left(X ; Y_{1}, Y_{2}\right) \\
& \rho_{1}(P) \triangleq \mathbb{E}\left[\mathrm{d}_{1}\left(X, Y_{1}\right)\right] \\
& \rho_{2}(P) \triangleq \mathbb{E}\left[\mathrm{d}_{2}\left(X, Y_{2}\right)\right]
\end{aligned}
$$

Lemma 3. The function $R_{2}\left(d_{1}, d_{2}, R_{1}\right)$ is non-increasing as a function of each argument when the others are kept fixed, jointly convex, and

$$
R_{2}\left(d_{1}, d_{2}, R_{1}\right)=\inf _{\substack{P: \\ I_{1}(P)=R_{1} \\ \rho_{1}(P)=d_{1} \\ \rho_{2}(P)=d_{2}}} I_{2}(P), \quad\left(d_{1}, d_{2}, R_{1}\right) \in \Omega,
$$

where the set $\Omega$ is defined in (22).

Proof. That $R_{2}\left(d_{1}, d_{2}, R_{1}\right)$ is non-increasing is obvious by definition. To show convexity, note first that since $u \log \frac{u}{v}$ is a convex function of $(u, v), D(P \| Q)$ is a convex function of $(P, Q)$, and so $I_{2}(P)$ is a convex function of $P$. Therefore, $I_{1}(P)$ is convex as a composition of a convex function $I_{2}(P)$ with an affine mapping $P \mapsto P_{Y_{1} \mid X}$.

Let the probability kernel $P_{a}$ attain $R_{2}\left(d_{1}^{a}, d_{2}^{a}, R_{1}^{a}\right)$ and $P_{b}$ attain $R_{2}\left(d_{1}^{b}, d_{2}^{b}, R_{1}^{b}\right)$. Let $P=\epsilon P_{a}+(1-\epsilon) P_{b}, d_{1}=\epsilon d_{1}^{a}+$ $(1-\epsilon) d_{1}^{b}, d_{2}=\epsilon d_{2}^{a}+(1-\epsilon) d_{2}^{b}, R_{1}=\epsilon R_{1}^{a}+(1-\epsilon) R_{1}^{b}$. Since $I_{1}(P)$ is convex and $\rho_{1}(P), \rho_{2}(P)$ are affine,

$$
\begin{aligned}
& I_{1}(P) \leq R_{1} \\
& \rho_{1}(P) \leq d_{1} \\
& \rho_{2}(P) \leq d_{2} .
\end{aligned}
$$

Furthermore, by convexity of $I_{2}(P)$,

$$
\begin{aligned}
I_{2}(P) \leq & \epsilon I_{2}\left(P_{a}\right)+(1-\epsilon) I_{2}\left(P_{b}\right) \\
= & \epsilon R_{2}\left(d_{1}^{a}, d_{2}^{a}, R_{1}^{a}\right) \\
& +(1-\epsilon) R_{2}\left(d_{1}^{b}, d_{2}^{b}, R_{1}^{b}\right) .
\end{aligned}
$$

Convexity of $R_{2}\left(d_{1}, d_{2}, R_{1}\right)$ follows by minimizing the left side of (122) over $P$ satisfying the constraints (118)-(120). To show (117), rewrite $R_{2}\left(d_{1}, d_{2}, R_{1}\right)$ as

$$
R_{2}\left(d_{1}, d_{2}, R_{1}\right)=\inf _{\substack{\tilde{R}_{1} \leq R \\ \tilde{d}_{1} \leq d_{1} \\ \tilde{d}_{2} \leq d_{2}}} \tilde{R}_{2}\left(\tilde{d}_{1}, \tilde{d}_{2}, \tilde{R}_{1}\right),
$$

where $\tilde{R}_{2}(\cdot, \cdot, \cdot)$ denotes the function in the right side of (117). Since for $\left(d_{1}, d_{2}, R_{1}\right) \in \Omega$, the function $R_{2}\left(d_{1}, d_{2}, R_{1}\right)$ is strictly decreasing in all arguments, the infimum (123) is achieved at the boundary, and (117) follows.

Put

$$
\begin{aligned}
& F\left(\nu_{1}, \lambda_{1}, \lambda_{2}\right) \triangleq \max \left\{h \geq 0: \forall\left(d_{1}, d_{2}, R_{2}\right)\right. \\
& \left.h-\lambda_{1} d_{1}-\lambda_{2} d_{2}-\nu_{1} R_{1} \leq R_{2}\left(d_{1}, d_{2}, R_{2}\right)\right\}
\end{aligned}
$$

i.e. $F\left(\nu_{1}, \lambda_{1}, \lambda_{2}\right)$ is the maximum of the $R_{2}$ axis intercepts of the hyperplanes $h-\lambda_{1} d_{1}-\lambda_{2} d_{2}-\nu_{1} R_{1}$ which have 
no point inside of the rate-distortion region in (18), i.e. for $\left(\lambda_{1}, \lambda_{2}, \nu_{1}\right) \geq 0$

$$
F\left(\nu_{1}, \lambda_{1}, \lambda_{2}\right) \triangleq \inf _{P_{Y_{1} \mid X}, P_{Y_{2} \mid X Y_{1}}} L\left(P_{Y_{1} \mid X}, P_{Y_{2} \mid X Y_{1}}\right),
$$

where

$$
\begin{aligned}
L\left(P_{Y_{1} \mid X}, P_{Y_{2} \mid X Y_{1}}\right) \triangleq & I\left(X ; Y_{1}, Y_{2}\right)+\nu_{1} I\left(X ; Y_{1}\right) \\
& +\lambda_{1} \mathbb{E}\left[\mathrm{d}_{1}\left(X, Y_{1}\right)\right]+\lambda_{2} \mathbb{E}\left[\mathrm{d}_{2}\left(X, Y_{2}\right)\right] .
\end{aligned}
$$

In other words, $L\left(P_{Y_{1} \mid X}, P_{Y_{2} \mid X Y_{1}}\right)$ is the Lagrangian and (125) is the Lagrangian dual problem.

Since $R_{2}\left(d_{1}, d_{2}, R_{1}\right)$ is convex and nonincreasing, to each $\left(d_{1}, d_{2}, R_{1}\right)$ such that $R_{2}\left(d_{1}, d_{2}, R_{1}\right)<\infty$, there exists $\left(\nu_{1}, \lambda_{1}, \lambda_{2}\right) \geq 0$ such that the hyperplane $h-\lambda_{1} d_{1}-\lambda_{2} d_{2}-$ $\nu_{1} R_{1}$ that passes through $\left(d_{1}, d_{2}, R_{1}\right)$ is tangent to the surface $R_{2}\left(d_{1}, d_{2}, R_{1}\right)$, and

$$
\begin{aligned}
& R_{2}\left(d_{1}, d_{2}, R_{1}\right) \\
= & \max _{\left(\nu_{1}, \lambda_{1}, \lambda_{2}\right) \geq 0}\left(F\left(\nu_{1}, \lambda_{1}, \lambda_{2}\right)-\nu_{1} R_{1}-\lambda_{1} d_{1}-\lambda_{2} d_{2}\right) .
\end{aligned}
$$

Theorem 2 is an immediate consequence of (126) and Theorem 5 below.

Theorem 5 (Necessary and sufficient conditions for an optimizer). In order for $\left(P_{Y_{1}^{*} \mid X}, P_{Y_{2}^{*} \mid X Y_{1}^{*}}\right)$ to achieve the infimum in (125), it is necessary and sufficient that

$$
\begin{aligned}
& \frac{d P_{X \mid Y_{1}^{*}=y_{1}}}{d P_{X}}(x)=\frac{\exp \left(-\frac{\lambda_{1}}{1+\nu_{1}} \mathrm{~d}_{1}\left(x, y_{1}\right)\right)}{\beta_{1}(x) \beta_{2}\left(x \mid y_{1}\right)^{-\frac{1}{1+\nu_{1}}}} \\
& \frac{d P_{X \mid Y_{1}^{*}=y_{1}, Y_{2}^{*}=y_{2}}}{d P_{X \mid Y_{1}^{*}=y_{1}}}=\frac{\exp \left(-\lambda_{2} \mathrm{~d}_{2}\left(x, y_{2}\right)\right)}{\beta_{2}\left(x \mid y_{1}\right)},
\end{aligned}
$$

where $0 \leq \beta_{1}(x) \leq 1,0 \leq \beta_{2}\left(x \mid y_{1}\right) \leq 1$ satisfy (32), (33).

Furthermore, the choice

$$
\begin{aligned}
\beta_{1}^{*}(x) & =\mathbb{E}\left[\beta_{2}^{*}\left(x \mid Y_{1}^{*}\right)^{\frac{1}{1+\nu_{1}}} \exp \left(-\frac{\lambda_{1}}{1+\nu_{1}} \mathrm{~d}_{1}\left(x, Y_{1}^{*}\right)\right)\right] \\
\beta_{2}^{*}\left(x \mid y_{1}\right) & =\mathbb{E}\left[\exp \left(-\lambda_{2} \mathrm{~d}_{2}\left(x, Y_{2}^{*}\right)\right) \mid Y_{1}^{*}=y_{1}\right]
\end{aligned}
$$

satisfies (32), (33), (127), (128).

Finally, for any $\beta_{1}(x) \geq 0, \beta_{2}\left(x \mid y_{1}\right) \geq 0$ satisfying (27), (28), we have for all $P_{Y_{1} Y_{2} \mid X}$

$$
L\left(P_{Y_{1} \mid X}, P_{Y_{2} \mid X Y_{1}}\right) \geq\left(1+\nu_{1}\right) \mathbb{E}\left[\log \frac{1}{\beta_{1}(X)}\right]
$$

with equality if and only if $\left(P_{Y_{1} \mid X}, P_{Y_{2} \mid X Y_{1}}\right)$ can be represented as in (127), (128), with the given $\left(\beta_{1}, \beta_{2}\right)$.

Proof of Theorem 5. The proof builds on the groundwork laid out in our proof of Theorem 4. In the first part of the proof, we will use the Donsker-Varadhan lemma and the assumption of the existence of optimizing kernels to characterize the optimal $\beta_{1}^{*}(x), \beta_{2}^{*}\left(x \mid y_{1}\right)$ as well as $P_{Y_{1}^{*} \mid X}$ and $P_{Y_{2}^{*} \mid X Y_{1}^{*}}$. We will apply the Donsker-Varadhan lemma twice, first for the second stage and then, thinking of the optimized rate at second stage as modifying the distortion measure at first stage, for the first stage. This reasoning, concluding at (141) below, will also ensure that equalities in (32) and (33) hold for $P_{Y_{1}^{*} Y_{2}^{*}-\text { a.e. }}$ $\left(y_{1}, y_{2}\right)$.

The second part of the proof, (142)-(166), shows the necessity of (32) and (33) for all $\left(y_{1}, y_{2}\right)$. This involves perturbing $P_{Y_{1}^{*} Y_{2}^{*}}$ by a delicately chosen auxiliary distribution and using the optimality of $P_{Y_{1}^{*} Y_{2}^{*}}$ to claim (32) and (33).

Having established these necessary conditions, we will proceed to show their sufficiency in the third and final part of the proof, (160)-(165).

First, we show that

$$
\inf _{P_{Y_{1} \mid X}, P_{Y_{2} \mid X Y_{1}}} L\left(P_{Y_{1} \mid X}, P_{Y_{2} \mid X Y_{1}}\right)=\mathbb{E}\left[\log \frac{1}{\beta_{1}^{*}(X)^{1+\nu_{1}}}\right] \text {. }
$$

For fixed probability kernels $P_{\bar{Y}_{1}}$ and $P_{\bar{Y}_{2} \mid \bar{Y}_{1}}$, consider the function

$$
\begin{aligned}
& \bar{L}\left(P_{Y_{1} \mid X}, P_{Y_{2} \mid X Y_{1}}, P_{\bar{Y}_{1}}, P_{\bar{Y}_{2} \mid \bar{Y}_{1}}\right) \\
& \triangleq \\
& \quad D\left(P_{Y_{2} \mid X Y_{1}} \| P_{\bar{Y}_{2} \mid \bar{Y}_{1}} \mid P_{X Y_{1}}\right)+\left(1+\nu_{1}\right) D\left(P_{Y_{1} \mid X} \| P_{\bar{Y}_{1}} \mid P_{X}\right) \\
& \quad+\lambda_{1} \mathbb{E}\left[\mathrm{d}_{1}\left(X, Y_{1}\right)\right]+\lambda_{2} \mathbb{E}\left[\mathrm{d}_{2}\left(X, Y_{2}\right)\right] .
\end{aligned}
$$

Since

$$
\begin{aligned}
& L\left(P_{Y_{1}^{*} \mid X}, P_{Y_{2}^{*} \mid X Y_{1}^{*}}\right) \\
= & \inf _{P_{Y_{1} \mid X}, P_{Y_{2} \mid X Y_{1}}, P_{\bar{Y}_{1}}, P_{\bar{Y}_{2} \mid \bar{Y}_{1}}} \bar{L}\left(P_{Y_{1} \mid X}, P_{Y_{2} \mid X Y_{1}}, P_{\bar{Y}_{1}}, P_{\bar{Y}_{2} \mid \bar{Y}_{1}}\right) \\
= & \bar{L}\left(P_{Y_{1}^{*} \mid X}, P_{Y_{2}^{*} \mid X Y_{1}^{*}}, P_{Y_{1}^{*}}, P_{Y_{2}^{*} \mid Y_{1}^{*}}\right)
\end{aligned}
$$

we have

$$
\bar{L}\left(P_{Y_{1} \mid X}, P_{Y_{2} \mid X Y_{1}}, P_{\bar{Y}_{1}}, P_{\bar{Y}_{2} \mid \bar{Y}_{1}}\right) \geq L\left(P_{Y_{1}^{*} \mid X}, P_{Y_{2}^{*} \mid X Y_{1}^{*}}\right),
$$

with equality if and only if $\left(P_{Y_{1} \mid X}, P_{Y_{2} \mid X Y_{1}}, P_{\bar{Y}_{1}}, P_{\bar{Y}_{2} \mid \bar{Y}_{1}}\right)=$ $\left(P_{Y_{1}^{*} \mid X}, P_{Y_{2}^{*} \mid X Y_{1}^{*}}, P_{Y_{1}^{*}}, P_{Y_{2}^{*} \mid Y_{1}^{*}}\right)$. Applying Lemma 1 twice, we compute the minimum of the left side of (136) particularized to $P_{\bar{Y}_{1}}=P_{Y_{1}^{*}}$ and $P_{\bar{Y}_{2} \mid \bar{Y}_{1}}=P_{Y_{2}^{*} \mid Y_{1}^{*}}$ :

$$
\begin{aligned}
& \bar{L}\left(P_{Y_{1} \mid X}, P_{Y_{2} \mid X Y_{1}}, P_{Y_{1}^{*}}, P_{Y_{2}^{*} \mid Y_{1}^{*}}\right) \\
\geq & \inf _{P_{Y_{1} \mid X}}\left\{\left(1+\nu_{1}\right) D\left(P_{Y_{1} \mid X} \| P_{Y_{1}^{*}} \mid P_{X}\right)+\lambda_{1} \mathbb{E}\left[\mathrm{d}_{1}\left(X, Y_{1}\right)\right]\right. \\
& \left.+\inf _{P_{Y_{2} \mid X Y_{1}}}\left\{D\left(P_{Y_{2} \mid X Y_{1}} \| P_{Y_{2}^{*} \mid Y_{1}^{*}} \mid P_{X Y_{1}}\right)+\lambda_{2} \mathbb{E}\left[\mathrm{d}_{2}\left(X, Y_{2}\right)\right]\right\}\right\} \\
= & \inf _{P_{Y_{1} \mid X}}\left\{\left(1+\nu_{1}\right) D\left(P_{Y_{1} \mid X}\left|P_{Y_{1}^{*}}\right| P_{X}\right)+\lambda_{1} \mathbb{E}\left[\mathrm{d}_{1}\left(X, Y_{1}\right)\right]\right. \\
& \left.+\mathbb{E}\left[\log \frac{1}{\beta_{2}^{*}\left(X \mid Y_{1}\right)}\right]\right\} \\
= & \left(1+\nu_{1}\right) \mathbb{E}\left[\log \frac{1}{\beta_{1}^{*}(X)}\right]
\end{aligned}
$$

where $\beta_{1}^{*}(x)$ and $\beta_{2}^{*}\left(x \mid y_{1}\right)$ are given in (129) and (130), respectively, and the optimizing $P_{Y_{1}^{*} \mid X}$ and $P_{Y_{2}^{*} \mid X Y_{1}^{*}}$ are specified in (127) and (128), letting $\beta_{1}(x)=\beta_{1}^{*}(x)$ and $\beta_{2}\left(x \mid y_{1}\right)=\beta_{2}^{*}\left(x \mid y_{1}\right)$ therein.

We proceed to show that $\beta_{1}^{*}(x)$ and $\beta_{2}^{*}\left(x \mid y_{1}\right)$ satisfy (32) and (33). For $P_{Y_{1}^{*}-\text { a.e. }} y_{1}$, we take expectations with respect to $P_{X}$ of both sides of (29) to conclude that

$$
\Sigma_{1}\left(y_{1}\right)=1 \text {. }
$$




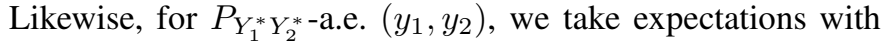
respect to $P_{X Y_{1}^{*}}$ of both sides of (30) to conclude that

$$
\Sigma_{2}\left(y_{1}, y_{2}\right)=1 \text {. }
$$

We next proceed to show that

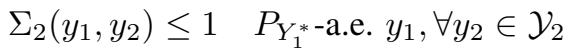

Particularizing the left side of (136) to $P_{Y_{1} \mid X}=P_{Y_{1}^{*} \mid X}$, $P_{\bar{Y}_{1}}=P_{Y_{1}^{*}}, P_{\bar{Y}_{2} \mid \bar{Y}_{1}}=P_{Y_{2}^{*} \mid Y_{1}^{*}}$ we apply Lemma 1 to characterize the minimum of the left side of (136) as

$$
\begin{gathered}
\bar{L}\left(P_{Y_{1}^{*} \mid X}, P_{Y_{2} \mid X Y_{1}^{*}}, P_{Y_{1}^{*}}, P_{Y_{2}^{*} \mid Y_{1}^{*}}\right) \\
\geq \quad\left(1+\nu_{1}\right) I\left(X ; Y_{1}^{*}\right)+\lambda_{1} \mathbb{E}\left[\mathrm{d}_{1}\left(X, Y_{1}^{*}\right)\right] \\
\quad \inf _{P_{Y_{2} \mid X Y_{1}^{*}}}\left\{D\left(P_{Y_{2} \mid X Y_{1}^{*}} \| P_{Y_{2}^{*} \mid Y_{1}^{*}} \mid P_{X Y_{1}^{*}}\right)\right. \\
\left.+\lambda_{2} \mathbb{E}\left[\mathrm{d}_{2}\left(X, Y_{2}\right)\right]\right\}
\end{gathered}
$$

To evaluate the infimum in (143), we apply Theorem 1 to

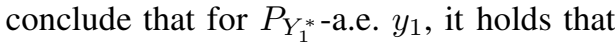

$$
\begin{aligned}
& D\left(P_{Y_{2} \mid X, Y_{1}^{*}=y_{1}} \| P_{Y_{2}^{*} \mid Y_{1}^{*}=y_{1}} \mid P_{X \mid Y_{1}^{*}=y_{1}}\right) \\
& +\lambda_{2} \mathbb{E}\left[\mathrm{d}_{2}\left(X, Y_{2}\right) \mid Y_{1}^{*}=y_{1}\right] \\
\geq \mathbb{E} & {\left[\log \frac{1}{\beta_{2}^{*}\left(X \mid y_{1}\right)} \mid Y_{1}^{*}=y_{1}\right] }
\end{aligned}
$$

with

$$
\mathbb{E}\left[\frac{\exp \left(-\lambda_{2} \mathrm{~d}_{2}\left(X, y_{2}\right)\right)}{\beta_{2}^{*}\left(X \mid y_{1}\right)} \mid Y_{1}^{*}=y_{1}\right] \leq 1 \quad P_{Y_{1}^{*}-\text { a.e. } y_{1}, \forall y_{2}}
$$

which, using (29), is equivalent to (142).

To finish the proof of (32) and (33), it remains to show that for all $y_{1}, y_{2}$ outside of the support of $P_{Y_{1}^{*} Y_{2}^{*}}$, (32) and (33) hold. Consider

$$
\begin{aligned}
& \bar{L}\left(P_{Y_{1} \mid X}, P_{Y_{2} \mid X Y_{1}}, P_{\bar{Y}_{1}}, P_{\bar{Y}_{2} \mid \bar{Y}_{1}}\right) \\
\geq & \inf _{P_{Y_{1} \mid X}}\left\{\left(1+\nu_{1}\right) D\left(P_{Y_{1} \mid X} \| P_{\bar{Y}_{1}} \mid P_{X}\right)+\lambda_{1} \mathbb{E}\left[\mathrm{d}_{1}\left(X, Y_{1}\right)\right]\right. \\
+ & \left.\inf _{P_{Y_{2} \mid X Y_{1}}}\left\{D\left(P_{Y_{2} \mid X Y_{1}} \| P_{\bar{Y}_{2} \mid \bar{Y}_{1}} \mid P_{X Y_{1}}\right)+\lambda_{2} \mathbb{E}\left[\mathrm{d}_{2}\left(X, Y_{2}\right)\right]\right\}\right\} \\
= & \inf _{P_{Y_{1} \mid X}}\left\{\left(1+\nu_{1}\right) D\left(P_{Y_{1} \mid X} \| P_{\bar{Y}_{1}} \mid P_{X}\right)+\lambda_{1} \mathbb{E}\left[\mathrm{d}_{1}\left(X, Y_{1}\right)\right]\right. \\
& \left.+\mathbb{E}\left[\log \frac{1}{\bar{\beta}_{2}\left(X \mid Y_{1}\right)}\right]\right\} \\
= & \left(1+\nu_{1}\right) \mathbb{E}\left[\log \frac{1}{\bar{\beta}_{1}(X)}\right]
\end{aligned}
$$

where

$$
\begin{aligned}
\bar{\beta}_{1}(x) & =\mathbb{E}\left[\bar{\beta}_{2}\left(x \mid \bar{Y}_{1}\right)^{\frac{1}{1+\nu_{1}}} \exp \left(-\frac{\lambda_{1}}{1+\nu_{1}} \mathrm{~d}_{1}\left(x, \bar{Y}_{1}\right)\right)\right] \\
\bar{\beta}_{2}\left(x \mid y_{1}\right) & =\mathbb{E}\left[\exp \left(-\lambda_{2} \mathrm{~d}_{2}\left(x, \bar{Y}_{2}\right)\right) \mid \bar{Y}_{1}=y_{1}\right] .
\end{aligned}
$$

Due to (136),

$$
\inf _{P_{\bar{Y}_{1}}, P_{\bar{Y}_{2} \mid \bar{Y}_{1}}} \mathbb{E}\left[\log \frac{1}{\bar{\beta}_{1}(X)}\right]=\mathbb{E}\left[\log \frac{1}{\beta_{1}^{*}(X)}\right]
$$

Now, we choose $P_{\bar{Y}_{1}}$ and $P_{\bar{Y}_{2} \mid \bar{Y}_{1}}$ (not independently of each other!) as

$$
P_{\bar{Y}_{1} \bar{Y}_{2}}\left(y_{1}, y_{2}\right)= \begin{cases}(1-\epsilon) P_{Y_{1}^{*} Y_{2}^{*}}\left(y_{1}, y_{2}\right) & \text { for } P_{Y_{1}^{*}-\text { a.e. }} y_{1} \\ \epsilon \delta_{y_{1}} P_{\bar{Y}_{2} \mid \bar{Y}_{1}=y_{1}}\left(y_{2}\right) & \text { otherwise }\end{cases}
$$

for some $0 \leq \epsilon \leq 1$, where $P_{\bar{Y}_{2} \mid \bar{Y}_{1}}$ is an arbitrary transition probability kernel.

With this choice,

$$
\begin{aligned}
\bar{\beta}_{1}(x)= & (1-\epsilon) \beta_{1}^{*}(x) \\
& +\epsilon \bar{\beta}_{2}\left(x \mid y_{1}\right)^{\frac{1}{1+\nu_{1}}} \exp \left(-\frac{\lambda_{1}}{1+\nu_{1}} \mathrm{~d}_{1}\left(x, y_{1}\right)\right) \\
\left.\bar{\beta}_{1}^{\prime}(x)\right|_{\epsilon=0}= & -\beta_{1}^{*}(x) \\
& +\bar{\beta}_{2}\left(x \mid y_{1}\right)^{\frac{1}{1+\nu_{1}}} \exp \left(-\frac{\lambda_{1}}{1+\nu_{1}} \mathrm{~d}_{1}\left(x, y_{1}\right)\right)
\end{aligned}
$$

Due to (150), the minimum of (148) is attained at $\epsilon=0$, so its right derivative with respect to $\epsilon$ evaluated at $\epsilon=0$ must be nonnegative:

$$
\begin{aligned}
& \left.\frac{\partial}{\partial \epsilon} \mathbb{E}\left[\log \frac{1}{\bar{\beta}_{1}(X)}\right]\right|_{\epsilon=0} \\
= & 1-\mathbb{E}\left[\frac{\bar{\beta}_{2}\left(X \mid y_{1}\right)^{\frac{1}{1+\nu_{1}}} \exp \left(-\frac{\lambda_{1}}{1+\nu_{1}} \mathrm{~d}_{1}\left(X, y_{1}\right)\right)}{\beta_{1}^{*}(X)}\right] \\
\geq & 0,
\end{aligned}
$$

and (33) follows by substituting $P_{\bar{Y}_{2} \mid \bar{Y}_{1}}=P_{Y_{2}^{*} \mid Y_{1}^{*}}$ in (155). Bringing the differentiation inside of the expectation is permitted by the dominated convergence theorem: the negative of the integrand in (154) is $\log ((1-\epsilon) a+\epsilon b)=\log (1-$ $\epsilon)+\log a+\log \left(1+\frac{\epsilon}{1-\epsilon} \frac{b}{a}\right)$, for some $a>0, b>0$, and the difference quotient of the last term is bounded as $0 \leq \frac{1}{\epsilon} \log \left(1+\frac{\epsilon}{1-\epsilon} \frac{b}{a}\right) \leq \frac{b}{a} \frac{\log e}{1-\delta}$, for all $0 \leq \epsilon \leq \delta<1$.

To show (32), notice that (155) implies that the necessary condition for $P_{Y_{1}^{*} \mid X}, P_{Y_{2}^{*} \mid X Y_{1}^{*}}$ to achieve the minimum is that (155) holds for all choices of the auxiliary kernel $P_{\bar{Y}_{2} \mid \bar{Y}_{1}}$, and so

$$
\sup _{P_{\bar{Y}_{2} \mid \bar{Y}_{1}}} \mathbb{E}\left[\frac{\bar{\beta}_{2}\left(X \mid y_{1}\right)^{\frac{1}{1+\nu_{1}}} \exp \left(-\frac{\lambda_{1}}{1+\nu_{1}} \mathrm{~d}_{1}\left(X, y_{1}\right)\right)}{\beta_{1}^{*}(X)}\right] \leq 1 .
$$

To simplify (156), we will find the conditions under which $P_{Y_{2}^{*} \mid Y_{1}^{*}=y_{1}}$ attains the supremum in the left side of (156). Put

$$
P_{\bar{Y}_{2} \mid \bar{Y}_{1}}=(1-\epsilon) P_{Y_{2}^{*} \mid Y_{1}^{*}}+\epsilon \delta_{y_{2}}
$$

With this choice,

$$
\begin{gathered}
\bar{\beta}_{2}\left(x \mid y_{1}\right)=(1-\epsilon) \beta_{2}^{*}\left(x \mid y_{1}\right)+\epsilon \exp \left(-\lambda_{2} \mathrm{~d}_{2}\left(x, y_{2}\right)\right) \\
\left.\frac{\partial}{\partial \epsilon} \log \bar{\beta}_{2}\left(x \mid y_{1}\right)\right|_{\epsilon=0}=-1+\frac{\exp \left(-\lambda_{2} \mathrm{~d}_{2}\left(x, y_{2}\right)\right)}{\beta_{2}^{*}\left(x \mid y_{1}\right)}
\end{gathered}
$$

The right derivative of the expression in the left side of (156) with respect to $\epsilon$ evaluated at $\epsilon=0$ is displayed in (166) below and is equivalent to (32). Note that bringing 
the differentiation inside of the expectation is allowed by the dominated convergence theorem: the difference quotient of the integrand in (156) is proportional to $\frac{((1-\epsilon) a+\epsilon b)^{\nu_{2}}-a^{\nu_{2}}}{\epsilon}$, for $a \geq 0, b \geq 0$, which is bounded below by 0 and above by a constant times $a^{\nu_{2}-1} b$ in the range $\epsilon \leq \delta<1$, for some $\delta$.

We proceed to show (131), which will imply the sufficiency part. We apply Theorem 1 twice to write

$$
\begin{aligned}
& L\left(P_{Y_{1} \mid X}, P_{Y_{2} \mid X Y_{1}}\right) \\
\geq & \left(1+\nu_{1}\right) I\left(X ; Y_{1}\right)+\lambda_{1} \mathbb{E}\left[\mathrm{d}_{1}\left(X, Y_{1}\right)\right] \\
& +I\left(X ; Y_{2} \mid Y_{1}\right)+\lambda_{2} \mathbb{E}\left[\mathrm{d}_{2}\left(X, Y_{2}\right)\right] \\
\geq & \left(1+\nu_{1}\right) I\left(X ; Y_{1}\right)+\lambda_{1} \mathbb{E}\left[\mathrm{d}_{1}\left(X, Y_{1}\right)\right]+\mathbb{E}\left[\log \frac{1}{\beta_{2}\left(X \mid Y_{1}\right)}\right]
\end{aligned}
$$$$
\geq\left(1+\nu_{1}\right) \mathbb{E}\left[\log \frac{1}{\beta_{1}(X)}\right]
$$

where (161) holds for all $\beta_{2}\left(x \mid y_{1}\right) \geq 0$ satisfying

$$
\mathbb{E}\left[\frac{\exp \left(-\lambda_{2} \mathrm{~d}_{2}\left(X, y_{2}\right)\right)}{\beta_{2}\left(X \mid y_{1}\right)} \mid Y_{1}=y_{1}\right] \leq 1 \forall\left(y_{1}, y_{2}\right) \in \mathcal{Y}_{1} \times \mathcal{Y}_{2}
$$

with equality if and only if $\left(P_{Y_{2} \mid X, Y_{1}=y_{1}}, \beta_{2}\right)=$ $\left(P_{Y_{2}^{*} \mid, X, Y_{1}^{*}=y_{1}}, \beta_{2}^{*}\right)$ for $P_{Y_{1}}$-a.e. $y_{1}$.

Likewise, (162) holds for all $\beta_{1}(x) \geq 0$ satisfying (28), with equality if and only if

$$
\begin{aligned}
\frac{d P_{X \mid Y_{1}=y_{1}}}{d P_{X}}(x) & =\frac{\beta_{2}\left(x \mid y_{1}\right)^{\frac{1}{1+\nu_{1}}} \exp \left(-\frac{\lambda_{1}}{1+\nu_{1}} \mathrm{~d}_{1}\left(x, y_{1}\right)\right)}{\beta_{1}(x)} \\
\beta_{1}(x) & =\mathbb{E}\left[\beta_{2}\left(x \mid Y_{1}\right)^{\frac{1}{1+\nu_{1}}} \exp \left(-\frac{\lambda_{1}}{1+\nu_{1}} \mathrm{~d}_{1}\left(x, Y_{1}\right)\right)\right] .
\end{aligned}
$$

Substituting (164) into (163), we obtain (27), and (131) follows, together with condition for equality.

$$
\mathbb{E}\left[\frac{\beta_{2}^{*}\left(X \mid y_{1}\right)^{\frac{1}{1+\nu_{1}}} \exp \left(-\frac{\lambda_{1}}{1+\nu_{1}} \mathrm{~d}_{1}\left(X, y_{1}\right)\right)}{\beta_{1}^{*}(X)}\left(1-\frac{\exp \left(-\lambda_{2} \mathrm{~d}_{2}\left(X, y_{2}\right)\right)}{\beta_{2}^{*}\left(X \mid y_{1}\right)}\right)\right] \geq 0
$$

\section{ITERATIVE ALGORITHM}

\section{A. Computation of single stage rate-distortion function}

In the context of finite source and reproduction alphabets, an algorithm for computation of rate-distortion functions was proposed by Blahut [8]. Below, we state it for general alphabets in Algorithm 1 and provide its convergence analysis in Theorem 6. In Section VI-B below, we generalize these results to successive refinement.

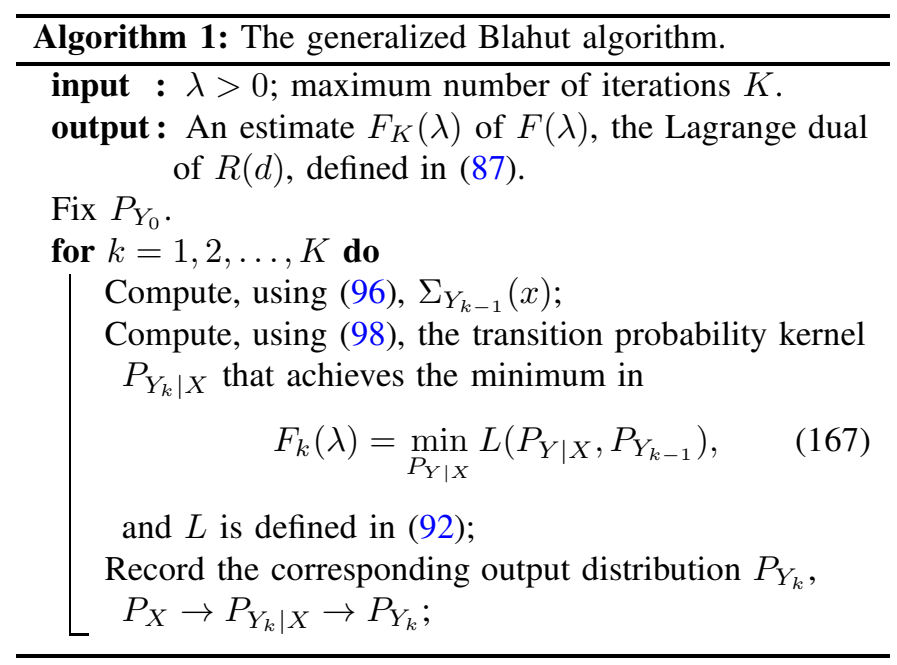

Theorem 6. Suppose $Y^{*}$ attains the minimum in (87), and let $Y_{0}$ be such that $D\left(Y^{*} \| Y_{0}\right)<\infty$. Consider Algorithm 1. The sequence $F_{k}(\lambda)$ is monotonically decreasing to $F(\lambda)$, and the convergence speed is bounded as

$$
F_{k}(\lambda)-F(\lambda) \leq \frac{D\left(Y^{*} \| Y_{0}\right)}{k}
$$

Proof. The analysis below is inspired by Csiszár [21]. From (93), we have

$$
\begin{aligned}
F_{k-1}(\lambda) & =L\left(P_{Y_{k} \mid X}, P_{Y_{k}}\right)+D\left(Y_{k} \| Y_{k-1}\right) \\
& \geq F_{k}(\lambda)+D\left(Y_{k} \| Y_{k-1}\right),
\end{aligned}
$$

and

$$
F_{k}(\lambda) \leq F_{k-1}(\lambda)
$$

with equality if and only if $Y_{k}=Y_{k-1}$, which implies that $F_{k-1}(\lambda)=F_{k}(\lambda)=F(\lambda)$.

Taking an expectation of (98) (particularized to $\bar{Y}=Y_{k-1}$ ) with respect to $P_{X Y^{*}}$, we conclude

$$
\begin{aligned}
F_{k}(\lambda) & =F(\lambda)+D\left(Y^{*} \| Y_{k-1}\right)-D\left(P_{Y^{*} \mid X} \| P_{Y_{k} \mid X} \mid P_{X}\right) \\
& \leq F(\lambda)+D\left(Y^{*} \| Y_{k-1}\right)-D\left(Y^{*} \| Y_{k}\right)
\end{aligned}
$$

where (173) holds by the data processing inequality for relative entropy. 
To show (168), we apply (171) and (173) as follows.

$$
\begin{aligned}
K F_{K}(\lambda)-K F(\lambda) & \leq \sum_{k=1}^{K} F_{k}(\lambda)-K F(\lambda) \\
& \leq \sum_{k=1}^{K}\left(D\left(Y^{*} \| Y_{k-1}\right)-D\left(Y^{*} \| Y_{k}\right)\right) \\
& =D\left(Y^{*} \| Y_{0}\right)-D\left(Y^{*} \| Y_{K}\right)
\end{aligned}
$$

Note that $D\left(Y^{*} \| Y_{0}\right)<\infty$ is a sufficient condition for convergence of Algorithm 1. This condition is trivially satisfied if the reproduction alphabet is finite and $P_{Y_{0}}$ is supported everywhere.

An alternative convergence guarantee can be obtained as follows. Considering (173) and noting that

$$
D\left(Y^{*} \| Y_{k-1}\right)-D\left(Y^{*} \| Y_{k}\right) \leq \sup _{y \in \mathcal{Y}} \log \frac{d P_{Y_{k}}}{d P_{Y_{k-1}}}(y),
$$

we can employ the following stopping criterion for the Blahut algorithm to guarantee estimation accuracy $\delta$ : if $\sup _{y \in \mathcal{Y}} \log \frac{d P_{Y_{k}}}{d P_{Y_{k-1}}}(y) \leq \delta$, then stop and output $\tilde{F}(\lambda)=$ $F_{k}(\lambda)$. If the same stopping rule is applied for all $\lambda \geq 0$, using (88), we find that the corresponding estimate of the ratedistortion function $\tilde{R}(d)$ satisfies the same accuracy guarantee:

$$
R(d) \leq \tilde{R}(d) \leq R(d)+\delta .
$$

\section{B. Computation of the rate-distortion function for successive} refinement

A generalization of discrete Blahut's algorithm to successive refinement is proposed in [3]. Algorithm 2 presents a generalization of the algorithm to abstract alphabets, and Theorem 7 presents its convergence analysis.

Theorem 7. Suppose $\left(P_{Y_{1}}^{*}, P_{Y_{2}^{*} \mid Y_{1}^{*}}\right)$ attain the minimum in (125), and let $P_{Y_{1}}^{0}$ and $P_{Y_{2} \mid Y_{1}}^{0}$ be such that $D\left(P_{Y_{1}^{*}} \| P_{Y_{1}}^{0}\right)<$ $\infty$ and $D\left(P_{Y_{2}^{*} \mid Y_{1}^{*}} \| P_{Y_{2} \mid Y_{1}}^{0} \mid P_{Y_{1}^{*}}\right)<\infty$. Consider Algorithm 2. The sequence $F_{k}\left(\nu_{1}, \lambda_{1}, \lambda_{2}\right)$ is monotonically decreasing to $F\left(\nu_{1}, \lambda_{1}, \lambda_{2}\right)$, and the convergence speed is bounded as

$$
\begin{aligned}
& F_{k}\left(\nu_{1}, \lambda_{1}, \lambda_{2}\right)-F\left(\nu_{1}, \lambda_{1}, \lambda_{2}\right) \\
\leq & \frac{1}{k}\left(\left(1+\nu_{1}\right) D\left(P_{Y_{1}^{*}} \| P_{Y_{1}}^{0}\right)+D\left(P_{Y_{2}^{*} \mid Y_{1}^{*}} \| P_{Y_{2} \mid Y_{1}}^{0} \mid P_{Y_{1}^{*}}\right)\right) .
\end{aligned}
$$

Proof. We build upon the ideas in the proof of Theorem 6. From the definition of $\bar{L}$ and $P_{Y_{1} \mid X}^{k}, P_{Y_{2} \mid X Y_{1}}^{k}$, we have

$$
\begin{aligned}
F_{k-1}= & \bar{L}\left(P_{Y_{1} \mid X}^{k}, P_{Y_{2} \mid X Y_{1}}^{k}, P_{Y_{1}}^{k-1}, P_{Y_{2} \mid Y_{1}}^{k-1}\right) \\
& +D\left(P_{Y_{2} \mid Y_{1}}^{k}\left\|P_{Y_{2} \mid Y_{1}}^{k-1}\right\| P_{Y_{1}}^{k}\right)+\left(1+\nu_{1}\right) D\left(P_{Y_{1}}^{k} \| P_{Y_{1}}^{k-1}\right) \\
\geq & F_{k} \\
& +D\left(P_{Y_{2} \mid Y_{1}}^{k}\left\|P_{Y_{2} \mid Y_{1}}^{k-1}\right\| P_{Y_{1}}^{k}\right)+\left(1+\nu_{1}\right) D\left(P_{Y_{1}}^{k} \| P_{Y_{1}}^{k-1}\right),
\end{aligned}
$$

where we suppressed the dependence of $F_{k}$ on $\left(\nu_{1}, \lambda_{1}, \lambda_{2}\right)$ for brevity, i.e. $F_{k}=F_{k}\left(\nu_{1}, \lambda_{1}, \lambda_{2}\right)$. It follows that

$$
F_{k} \leq F_{k-1} \text {, }
$$

Algorithm 2: The generalized Blahut algorithm for successive refinement.

input : $\left(\nu_{1}, \lambda_{1}, \lambda_{2}\right)>0$; maximum number of iterations $K$.

output: An estimate $F_{K}\left(\nu_{1}, \lambda_{1}, \lambda_{2}\right)$ of $F\left(\nu_{1}, \lambda_{1}, \lambda_{2}\right)$, the Lagrange dual of $R_{2}\left(d_{1}, d_{2}, R_{1}\right)$, defined in (125).

Fix $P_{Y_{1}}^{0}$ and $P_{Y_{2} \mid Y_{1}}^{0}$;

for $k=1,2, \ldots, K$ do

Compute

$$
\begin{aligned}
& \beta_{2}^{k-1}\left(x \mid y_{1}\right) \\
= & \mathbb{E}_{P_{Y_{2} \mid Y_{1}=y_{1}}^{k-1}}\left[\exp \left(-\lambda_{2} \mathrm{~d}_{2}\left(x, Y_{2}\right)\right) \mid Y_{1}=y_{1}\right] \\
& \beta_{1}^{k-1}(x) \\
= & \mathbb{E}_{P_{Y_{1}}^{k-1}}\left[\beta_{2}^{k-1}\left(x \mid Y_{1}\right)^{\frac{1}{1+\nu_{1}}} \exp \left(-\frac{\lambda_{1}}{1+\nu_{1}} \mathrm{~d}_{1}\left(x, Y_{1}\right)\right)\right] ;
\end{aligned}
$$

Using

$$
\begin{aligned}
& \frac{d P_{Y_{1} \mid X=x}^{k}\left(y_{1}\right)}{d P_{Y_{1}}^{k-1}\left(y_{1}\right)}=\frac{\exp \left(-\frac{\lambda_{1}}{1+\nu_{1}} \mathrm{~d}_{1}\left(x, y_{1}\right)\right)}{\beta_{1}^{k-1}(x) \beta_{2}^{k-1}\left(x \mid y_{1}\right)^{-\frac{1}{1+\nu_{1}}}}, \\
& \frac{d P_{Y_{2} \mid X=x, Y_{1}=y_{1}}^{k}\left(y_{2}\right)}{d P_{Y_{2} \mid Y_{1}=y_{1}}^{k-1}\left(y_{2}\right)}=\frac{\exp \left(-\lambda_{2} \mathrm{~d}_{2}\left(x, y_{2}\right)\right)}{\beta_{2}^{k-1}\left(x \mid y_{1}\right)},
\end{aligned}
$$

compute the transition probability kernels $P_{Y_{1} \mid X}^{k}$ and $P_{Y_{2} \mid X Y_{1}}^{k}$ that achieve the minimum in

$$
\begin{aligned}
& F_{k}\left(\nu_{1}, \lambda_{1}, \lambda_{2}\right) \\
& =\min _{P_{Y_{1} \mid X}, P_{Y_{2} \mid X Y_{1}}} \bar{L}\left(P_{Y_{1} \mid X}, P_{Y_{2} \mid X Y_{1}}, P_{Y_{1}}^{k-1}, P_{Y_{2} \mid Y_{1}}^{k-1}\right) \\
& =\left(1+\nu_{1}\right) \mathbb{E}\left[\log \frac{1}{\beta_{1}^{k-1}(X)}\right]
\end{aligned}
$$

where $\bar{L}$ is defined in (133), and the minimum is computed in (148). Compute the corresponding $P_{Y_{1}}^{k}$ and $P_{Y_{2} \mid Y_{1}}^{k}$;

with equality if and only if $P_{Y_{2} \mid Y_{1}}^{k-1} P_{Y_{1}}^{k-1}=P_{Y_{2} \mid Y_{1}}^{k} P_{Y_{1}}^{k}$, which implies that $F_{k-1}=F_{k}=F$.

Taking expectations of the logarithms of (181) and (182) with respect to $P_{X Y_{1}^{*} Y_{2}^{*}}$ and using (148), we deduce that

$$
\begin{aligned}
& F_{k} \\
= & F+\left(1+\nu_{1}\right)\left(D\left(P_{Y_{1}^{*}} \| P_{Y_{1}}^{k-1}\right)-D\left(P_{Y_{1}^{*} \mid X}\left\|P_{Y_{1}^{k} \mid X}\right\| P_{X}\right)\right) \\
& +D\left(P_{Y_{2}^{*} \mid Y_{1}^{*}} \| P_{Y_{2} \mid Y_{1}}^{k-1} \mid P_{Y_{1}^{*}}\right) \\
& -D\left(P_{Y_{2}^{*} \mid X_{1} Y_{1}^{*}} \| P_{Y_{2} \mid X_{1} Y_{1}}^{k} \mid P_{X_{1} Y_{1}^{*}}\right) \\
\leq & F+\left(1+\nu_{1}\right)\left(D\left(P_{Y_{1}^{*}} \| P_{Y_{1}}^{k-1}\right)-D\left(P_{Y_{1}^{*}} \| P_{Y_{1}}^{k}\right)\right) \\
& +D\left(P_{Y_{2}^{*} \mid Y_{1}^{*}} \| P_{Y_{2} \mid Y_{1}}^{k-1} \mid P_{Y_{1}^{*}}\right)-D\left(P_{Y_{2}^{*} \mid Y_{1}^{*}} \| P_{Y_{2} \mid Y_{1}}^{k} \mid P_{Y_{1}^{*}}\right)
\end{aligned}
$$

where (190) holds by the data processing inequality for relative entropy. 
To show (185), we apply (188) and (190) as follows.

$$
\begin{aligned}
& K F_{K}-K F \\
\leq & \sum_{k=1}^{K} F_{k}-K F \\
\leq & \sum_{k=1}^{K}\left[\left(1+\nu_{1}\right)\left(D\left(P_{Y_{1}^{*}} \| P_{Y_{1}}^{k-1}\right)-D\left(P_{Y_{1}^{*} \mid X} \| P_{Y_{1}}^{k}\right)\right)\right. \\
& \left.+D\left(P_{Y_{2}^{*} \mid Y_{1}^{*}} \| P_{Y_{2} \mid Y_{1}}^{k-1} \mid P_{Y_{1}^{*}}\right)-D\left(P_{Y_{2}^{*} \mid Y_{1}^{*}} \| P_{Y_{2} \mid Y_{1}}^{k} \mid P_{Y_{1}^{*}}\right)\right] \\
= & \left(1+\nu_{1}\right)\left(D\left(P_{Y_{1}^{*}} \| P_{Y_{1}}^{0}\right)-D\left(P_{Y_{1}^{*}} \| P_{Y_{1}}^{K}\right)\right) \\
& +D\left(P_{Y_{2}^{*} \mid Y_{1}^{*}} \| P_{Y_{2} \mid Y_{1}}^{0} \mid P_{Y_{1}^{*}}\right)-D\left(P_{Y_{2}^{*} \mid Y_{1}^{*}} \| P_{Y_{2} \mid Y_{1}}^{K} \mid P_{Y_{1}^{*}}\right)
\end{aligned}
$$

Using (190), we can obtain the following analog of the stopping criterion in (177): to achieve accuracy $F_{k}\left(\nu_{1}, \lambda_{1}, \lambda_{2}\right)-$ $F\left(\nu_{1}, \lambda_{1}, \lambda_{2}\right) \leq \delta$, stop as soon as

$$
\sup _{\left(y_{1}, y_{2}\right) \in \mathcal{Y}_{1} \times \mathcal{Y}_{2}}\left(1+\nu_{1}\right) \log \frac{d P_{Y_{1}}^{k}}{d P_{Y_{1}}^{k-1}}(y)+\frac{d P_{Y_{2} \mid Y_{1}}^{k}}{d P_{Y_{2} \mid Y_{1}}^{k-1}}(y) \leq \delta .
$$

For finite alphabet sources, a counterpart of (194) was proposed by Tuncel and Rose [3].

\section{Numerical example}

Consider successive refinement of $X \sim \mathcal{N}(0,1)$ under squared error distortion. As is well known, Gaussian source under squared distortion is successively refinable [18], so at any $0<d_{2} \leq d_{1} \leq 1$ and $R\left(d_{1}\right) \leq R_{1}, R_{2}\left(d_{1}, d_{2}, R_{1}\right)=$ $R\left(d_{2}\right)=\frac{1}{2} \log \frac{1}{d_{2}}$.

In this experiment, we ran Algorithm 2 to verify that it computes an estimate of $R_{2}\left(d_{1}, d_{2}, R_{1}\right)$ that closely matches $R\left(d_{2}\right)$.

We fixed $\lambda_{1}=5 / 9$, which corresponds to $d_{1}=0.9$. We also fixed $\nu_{1}=1$ (for this example, the choice of $\nu_{1}>0$ is immaterial and can be chosen arbitrarily, as per discussion after (39)). We set starting densities $P_{Y_{1}}^{0}$ and $P_{Y_{2} \mid Y_{1}=y_{1}}^{0}$ to be $\mathcal{N}(0,1)$ and $\mathcal{N}\left(y_{1}, 1\right)$, respectively, ensuring that all the densities in Algorithm 2 are Gaussian, and all the integrals can be computed in closed form. We chose 31 exponentially spaced slope samples $\lambda_{2}>0$, and we ran the algorithm for the maximum of $K=20$ iterations at each choice of $\lambda_{2}$. In Fig. 1, 31 straight lines of slopes $-\lambda_{2}$ correspond to $F_{K}-\lambda_{2} d_{2}-\lambda_{1} d_{1}-\nu_{1} R_{1}$. Their upper convex envelope is the numerical estimate of $R_{2}\left(d_{1}, d_{2}, R_{1}\right)$ according to the algorithm. In Fig. 3, it is undistinguishable from the the thick curve, which represents the theoretical minimum total rate, $\frac{1}{2} \log \frac{1}{d_{2}}$.

Computing the expectations in Algorithms 1 and 2 is easy to do if the output alphabets are finite, even if $P_{X}$ is continuous, a case also not previously addressed in literature. For infinite output alphabets, computing these expectations can be a computational bottleneck. Still, one could use Algorithms 1 and 2 to look for the best approximation within a certain family of distributions parametrized by a finite number of parameters. The quality of the approximation will depend on how appropriately the parametric family is chosen. To choose a good family, one could look for a separate theoretical argument that would ensure that the infimum is attained within some class of distributions. Theorems 6 and 7 would then ensure convergence when running the algorithm within that class.

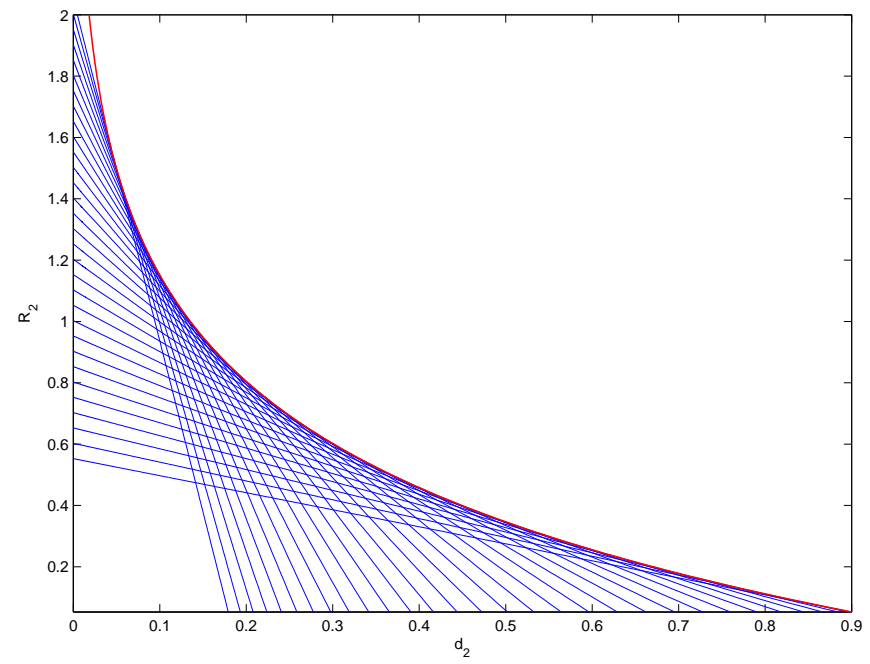

Fig. 3. The minimum total rate at stage 2 for Gaussian successive refinement, for fixed $\lambda_{1}=5 / 9$ (corresponding to $d_{1}=0.9, R_{1}=-.5 \log .9 \approx .05$ ).

\section{CONCLUSION}

In this paper, we revisited the parametric representation of rate-distortion function of abstract sources (Theorem 1, proof in Section IV). We showed its generalization to the successive refinement problem (Theorem 2, proof in Section V). That representation leads to a tight nonasymptotic converse bound for successive refinement, presented in Section III. It also helps to formulate and prove the convergence of an iterative algorithm that can be applied to compute the rate-distortion function on abstract alphabets, presented in Section VI.

It will be interesting to see whether the approach presented in this paper can be applied to study rate-distortion regions of other important multiterminal information theory problems, such as lossy compression with side information available at decoder (the Wyner-Ziv problem [22]), the multiple descriptions problem [23] and lossy compression with possibly absent side information (the Kaspi problem [24]). It also paves the way to a refined nonasymptotic analysis of successive refinement for abstract sources.

\section{ACKNOWLEDGEMENT}

We would like to thank Lin Zhou for valuable comments regarding a second-order analysis of the nonasymptotic bounds in Section III, and both anonymous reviewers for detailed suggestions.

\section{REFERENCES}

[1] V. Kostina and E. Tuncel, "The rate-distortion function for successive refinement of abstract sources," in Proceedings 2017 IEEE International Symposium on Information Theory, Aachen, Germany, June 2017, pp. 1923-1927. 
[2] I. Csiszár, "On an extremum problem of information theory," Studia Scientiarum Mathematicarum Hungarica, vol. 9, no. 1, pp. 57-71, Jan. 1974.

[3] E. Tuncel and K. Rose, "Computation and analysis of the n-layer scalable rate-distortion function," IEEE Transactions on Information Theory, vol. 49, no. 5, pp. 1218-1230, 2003.

[4] C. E. Shannon, "Coding theorems for a discrete source with a fidelity criterion," IRE Int. Conv. Rec., vol. 7, no. 1, pp. 142-163, Mar. 1959, reprinted with changes in Information and Decision Processes, R. E. Machol, Ed. New York: McGraw-Hill, 1960, pp. 93-126.

[5] R. Gallager, Information theory and reliable communication. John Wiley \& Sons, Inc. New York, 1968.

[6] T. Berger, Rate distortion theory. Prentice-Hall, Englewood Cliffs, NJ, 1971.

[7] I. Csiszár and J. Körner, Information Theory: Coding Theorems for Discrete Memoryless Systems, 2nd ed. Cambridge Univ Press, 2011.

[8] R. Blahut, "Computation of channel capacity and rate-distortion functions," IEEE Transactions on Information Theory, vol. 18, no. 4, pp. 460-473, Jul. 1972.

[9] V. Kostina, "When is Shannon's lower bound tight?" in Proceedings 54th Annual Allerton Conference on Communication, Control and Computing, Monticello, IL, Oct. 2016, pp. 982-989.

[10] - "Data compression with low distortion and finite blocklength," IEEE Transactions on Information Theory, vol. 63, no. 7, pp. 42684285, July 2017.

[11] V. Kostina and S. Verdú, "Fixed-length lossy compression in the finite blocklength regime," IEEE Transactions on Information Theory, vol. 58, no. 6, pp. 3309-3338, June 2012.

[12] A. No, A. Ingber, and T. Weissman, "Strong successive refinability and rate-distortion-complexity tradeoff," IEEE Transactions on Information Theory, vol. 62, no. 6, pp. 3618-3635, 2016.

[13] L. Zhou, V. Y. Tan, and M. Motani, "Second-order and moderate deviations asymptotics for successive refinement," IEEE Transactions on Information Theory, vol. 63, no. 5, pp. 2896-2921, 2017.

[14] C.-I. Chang and L. D. Davisson, "On calculating the capacity of an infinite-input finite (infinite)-output channel," IEEE Transactions on Information Theory, vol. 34, no. 5, pp. 1004-1010, Sep 1988.

[15] K. Rose, "A mapping approach to rate-distortion computation and analysis," IEEE Transactions on Information Theory, vol. 40, no. 6, pp 1939-1952, Nov 1994.

[16] B. Rimoldi, "Successive refinement of information: Characterization of the achievable rates," IEEE Transactions on Information Theory, vol. 40, no. 1, pp. 253-259, 1994.

[17] M. Effros, "Distortion-rate bounds for fixed-and variable-rate multiresolution source codes," IEEE Transactions on Information Theory, vol. 45, no. 6, pp. 1887-1910, 1999.

[18] W. H. Equitz and T. M. Cover, "Successive refinement of information," IEEE Transactions on Information Theory, vol. 37, no. 2, pp. 269-275, 1991.

[19] M. D. Donsker and S. R. S. Varadhan, "Asymptotic evaluation of certain markov process expectations for large time, I," Communications on Pure and Applied Mathematics, vol. 28, no. 1, pp. 1-47, 1975.

[20] Y. Polyanskiy, "Information theory lecture notes," Dep. Electrical Engineering and Computer Science, M.I.T., 2012.

[21] I. Csiszár, "On the computation of rate-distortion functions (corresp.)," IEEE Transactions on Information Theory, vol. 20, no. 1, pp. 122-124, 1974.

[22] A. D. Wyner and J. Ziv, "The rate-distortion function for source coding with side information at the decoder," IEEE Transactions on Information Theory, vol. 22, no. 1, pp. 1-10, 1976.

[23] J. K. Wolf, A. D. Wyner, and J. Ziv, "Source coding for multiple descriptions," Bell Labs Technical Journal, vol. 59, no. 8, pp. 1417 1426, 1980.

[24] A. H. Kaspi, "Rate-distortion function when side-information may be present at the decoder," IEEE Transactions on Information Theory, vol. 40, no. 6, pp. 2031-2034, 1994. 